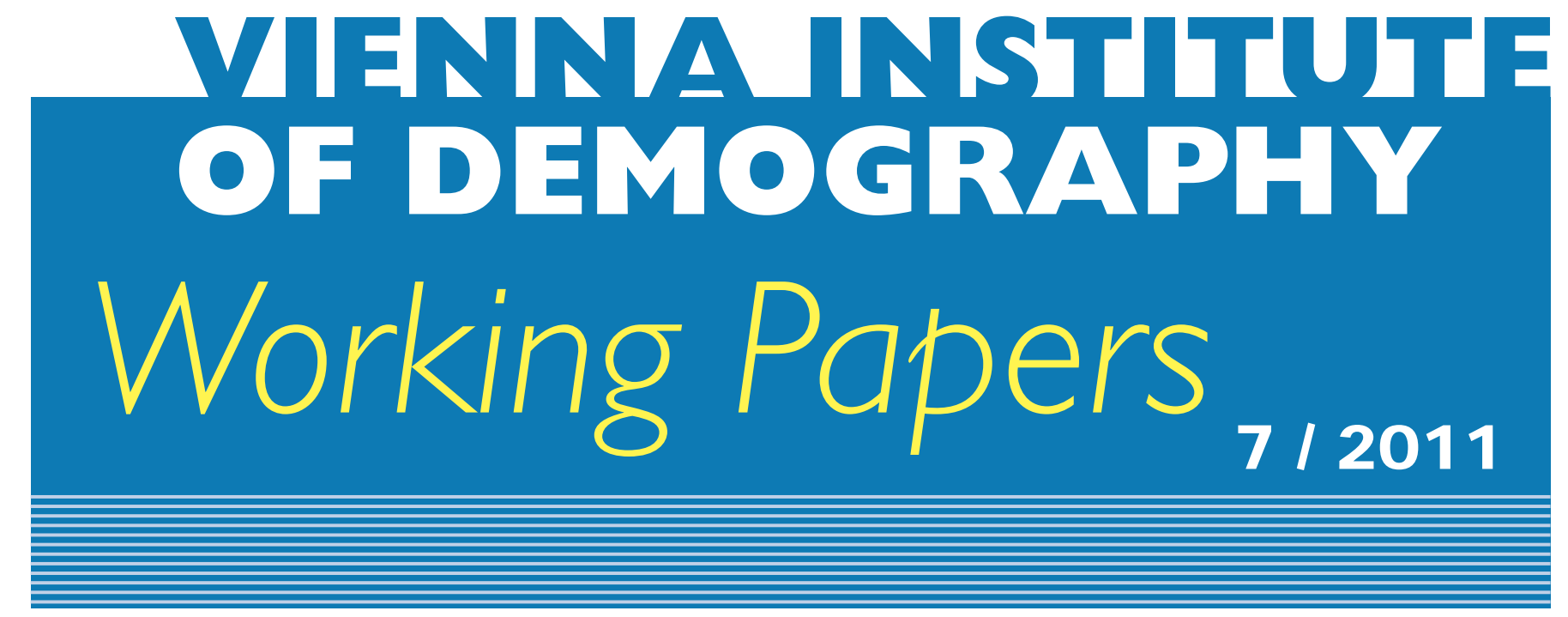

Kryštof Zeman, Tomáš Sobotka, Richard Gisser, M aria W inkler-Dworak, and W olfgang Lutz

\title{
Geburtenbarometer Wien: Analyse der konvergenten Fertilität zwischen Wien und Ö sterreich
}

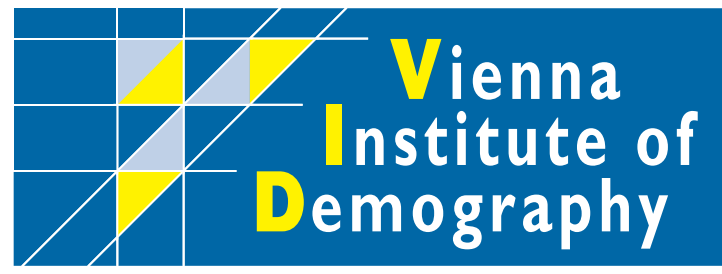

Vienna Institute of Demography Austrian Academy of Sciences

Wohllebengasse 12-14

A-I040 Vienna $\cdot$ Austria

E-Mail:vid@oeaw.ac.at

Website: www.oeaw.ac.at/vid

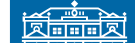

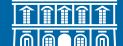

OAW

Austrian Academy of Sciences 


\section{Kurzbeschreibung}

Das Geburtenbarometer Wien zielt darauf ab, ein aktuelles Fertilitätsmonitoring für Wien zur Verfügung zu stellen, basierend auf einem Satz jährlicher und vierteljährlicher Fruchtbarkeitsindikatoren und regelmäßigen zusammenfassenden Berichten. Die vorliegende Studie informiert über die verwendeten Daten, Methoden und Indikatoren und kontrastiert die Hauptresultate mit Daten für die anderen österreichischen Regionen und für das ganze Land. Den größten Teil des 20. Jahrhunderts hindurch lag die Fertilität in Wien weit unterhalb jener des übrigen Österreichs. Weil sich die Fruchtbarkeit in Wien deutlich zwischen den in Österreich und den im Ausland geborenen Frauen unterscheidet, werden diese Fertilitätsdifferentiale und ihr Einfluss auf die gesamten Fertilitätsmuster Wiens und Österreichs betrachtet. Zugewanderte Frauen hatten in Wien seit der Mitte der 1980er Jahre nicht nur einen schnell zunehmenden Anteil an den Gesamtgeburten und trugen folglich zu einem allmählichen Anstieg der absoluten Geburtenzahl in der Stadt bei, sondern ihre höheren Fruchtbarkeitsziffern halfen auch, die Periodenfertilitätsraten in Richtung auf die Niveaus zu bringen, die in anderen Regionen Österreichs verzeichnet werden. Die vorliegende Studie veranschaulicht die ausgeprägte Wiener Bevölkerungsdynamik, in der sich eine hohe Kinderlosigkeit und niedrige Fertilität mit einer positiven Bilanz zwischen Geburten und Sterbefällen sowie anhaltendem Bevölkerungszuwachs verbinden und die Bevölkerungstrends stark durch die direkten und indirekten Auswirkungen der Migration beeinflusst werden.

\section{Schlüsselwörter}

Fertilität, Wien, Österreich, Familienzuwachswahrscheinlichkeiten, Fertilitätsmessung, Fertilität von Migrantinnen

\section{Autoren}

Kryštof Zeman ist Research Scientist am Vienna Institute of Demography (VID), Österreichische Akademie der Wissenschaften (ÖAW). Email: krystof.zeman@oeaw.ac.at

Tomáš Sobotka ist Research Scientist am VID, ÖAW. Email: tomas.sobotka@oeaw.ac.at Richard Gisser ist Stellvertretender Direktor des am VID, ÖAW.

Maria Winkler-Dworak ist Research Scientist am am VID, ÖAW.

Wolfgang Lutz ist Direktor des VID, ÖAW; Leader des World Population Program, Internationales Institut for Angewandte Systemanalyse, Laxenburg, Österreich; Professor für Sozial- und Wirtschaftsstatistik, Wirtschaftsuniversität Wien; und Direktor des Wittgenstein Centre for Demography and Global Human Capital.

\section{Danksagungen}

Der Kulturabteilung der Stadt Wien (MA 7, GZ: MA 7 - 2819/08) wird gedankt für die finanzielle Unterstützung, die zur Einrichtung des Geburtenbarometers Wien beitrug. Das Referat für Statistiken und Analysen der Stadt Wien (MA 5) stellte dankenswerterweise einige Bevölkerungsdaten bereit. Das Geburtenbarometer-Projekt wurde durch eine Förderung des Bundesministeriums für Soziale Sicherheit, Generationen und Konsumentenschutz, GZ: BMSG-442030/006-V7/2004, ermöglicht. Der Bundesanstalt Statistik Österreich (Statistik Austria) gebührt besonderer Dank für die fortgesetzte Zurverfügungstellung von Geburtendaten und Bevölkerungsstatistiken, die für die Aktualisierung des Geburtenbarometers notwendig sind. 


\title{
Geburtenbarometer Wien: Analyse der konvergenten Fertilität zwi- schen Wien und Österreich
}

\author{
Kryštof Zeman, Tomáš Sobotka, Richard Gisser, Maria Winkler-Dworak und \\ Wolfgang Lutz
}

\section{Hintergrund}

Im Jahr 2004 schlug das Institut für Demographie der Österreichischen Akademie der Wissenschaften (Vienna Institute of Demography - VID) die Einrichtung eines „Geburtenbarometer“ genannten Fertilitätsmonitors für Österreich vor (Sobotka et al. 2005), dessen Hauptziel darin liegt, eine kontinuierliche Beobachtung der Periodenfertilitätstrends nach den neuesten Methoden zu liefern. Dieser regelmäßig aktualisierte Monitor erlaubt ein viel besseres Verständnis der Fertilitätsentwicklungen, als es mit Hilfe von jährlichen Indizes der Fruchtbarkeitsziffern möglich wäre, vor allem mit der bisher am häufigsten benutzten Perioden-Gesamtfruchtbarkeitsziffer (Total Fertility Rate, TFR). Insbesondere liefert das Geburtenbarometer eine Reihe von alters-, dauer- und paritätsspezifischen Indizes der Fertilität, die anzeigen, ob eine Änderung im Fertilitätstrend über die Zeit auf sich ändernde Erstgeburtenziffern oder auf geänderte Übergangsraten zu Geburten zweiter oder höherer Ordnung zurückzuführen ist. Diese paritätsspezifischen Fruchtbarkeitsziffern ergeben einen zusammenfassenden Index der Gesamtfertilität, die Durchschnittliche Perioden-Parität (Period Average Parity, PAP), die neben den herkömmlichen Gesamtfruchtbarkeitsziffern berechnet und veröffentlicht wird. Die PAP hat zwei Hauptvorteile gegenüber der gewöhnlichen TFR. Zunächst basiert sie auf paritätsspezifischen Indikatoren; folglich berücksichtigt sie die aktuelle Paritätsverteilung in der weiblichen Bevölkerung, was bei der TFR nicht der Fall ist, welche die Fertilität nur hinsichtlich der Altersverteilung der Frauen im reproduktiven Alter standardisiert. Zweitens ist die PAP weniger durch die laufenden Änderungen im Geburtentiming beeinflusst (,Tempo-Effekte“; Bongaarts und Feeney 1998), derentwegen die TFR in Österreich seit den siebziger Jahren verzerrt wird. Infolgedessen reflektiert die PAP das tatsächliche Fertilitätsniveau (Quantum) in Österreich besser, und sie liegt auch näher bei der abgeschlossenen Fruchtbarkeit der Geburtskohorten der Frauen, die während eines gegebenen Zeitraums Kinder bekommen. Schließlich stellt das Geburtenbarometer für Österreich Zeitreihen von Fruchtbarkeitsziffern auf Monatsbasis zur Verfügung und ermöglicht es damit, neueste Fertilitätstendenzen zu studieren sowie Fertilitätsreaktionen auf neu eingeführte familienpolitische Maßnahmen und ausgewählte sozioökonomische Trends zu analysieren.

Die erfolgreiche Installierung des österreichischen Geburtenbarometers hat auch zu einer stärkeren Verbreitung alternativer Fertilitätsindikatoren geführt, die eine willkommene Alternative zur Perioden-TFR bieten, die zunehmend als problematischer und eventuell sogar irreführender Fruchtbarkeitsindikator empfunden wird (Sobotka und Lutz 2009). ${ }^{1}$ Von Anfang an wurde die Einrichtung eines ähnlichen Monitors in anderen Ländern und auch in kleineren regionalen Einheiten erwogen. Die Unterstützung seitens der Stadt Wien im Rahmen der Wissenschaftsförderung bietet eine Gelegenheit, das „Geburtenbarometer Wien“ zu erstellen und einzigartige Einblicke in Familienbildungsmuster und Fertilitätstrends in dieser Stadt zu gewinnen, die bis vor kurzem die niedrigsten Fruchtbarkeitsziffern in Österreich aufwies. Es ist zu hoffen, dass das Geburtenbarometer Wien

\footnotetext{
${ }^{1}$ Das Geburtenbarometer für Österreich findet sich auf der Website des Vienna Institute of Demography (VID); siehe http://www.oeaw.ac.at/vid/barometer/.
} 
eine intensiv genutzte Quelle von Fertilitätsdaten werden, interessante Analysen anregen und zu einer weiteren Verbreitung des Geburtenbarometers beitragen wird. Wie im Fall des Geburtenmonitors für Österreich wird das Geburtenbarometer Wien sowohl kurzfristige (vierteljährliche) als auch jährliche Resultate in Form knapper Berichte und einer regelmäßig aktualisierten Datenbank zur Verfügung stellen, die allen interessierten Benutzern zugänglich sind.

\section{Einleitung: Warum Fertilitätsmonitoring für Wien?}

Die Stadt Wien präsentiert auf ihren Werbeunterlagen, Broschüren und Plakaten stolz ein Logo mit der Feststellung „Wien ist anders“. Während diese Aussage nicht für alle Aspekte des Soziallebens zutreffend sein dürfte, ist sie demographisch passend, besonders in Bezug auf Bevölkerungsstruktur, Migration und Fruchtbarkeitsziffern. Im Vergleich zum übrigen Österreich ist Wien, mit einer Bevölkerung von 1,71 Millionen (2010), eine Region mit besonders starker Zuwanderung aus dem Ausland. Nach der letzten Volkszählung von 2001 waren 31\% der Wiener Bevölkerung im Ausland geboren, weit über dem entsprechenden Anteil für Österreich (14\%). Wien zeigt auch ein ausgeprägtes Fertilitätsmuster, das sich jedoch im Lauf der Zeit geändert hat. In der Vergangenheit waren, ähnlich wie bei anderen Großstädten Europas, die Fruchtbarkeitsziffern in Wien weit unter denen, die in anderen Regionen Österreichs verzeichnet wurden. 1934, auf dem Höhepunkt der ökonomischen Rezession der Dreißigerjahre erreichte die Perioden-TFR ein extrem niedriges Niveau von 0,61 (Gisser et al. 1975: 104, Tabelle 39). Auch während der Nachkriegszeit blieb die Gesamtfruchtbarkeitsziffer in Wien unter 2 und folglich unterhalb des Bevölkerungsersatzniveaus, ${ }^{2}$ sogar während der Zeit des Babybooms, der die TFR in Österreich 1963 auf 2,82 hinaufdrückte. Wegen der hartnäckig niedrigen Fruchtbarkeitsziffern hatte Wien auch bis vor kurzem eine negative Bilanz zwischen Geburten und Todesfällen aufzuweisen, die von der Mitte der 1920er Jahre bis 2004 vorherrschte (Statistik Austria 2009a). Die lange Dauer der niedrigen Fertilitätsraten hatte zu einem ungewöhnlich schnellen und frühen „Ergrauen“ der Wiener Bevölkerung geführt, wobei der Anteil der Bevölkerung von 60 und mehr Jahren bereits 1970 bei 28\% kulminierte (Lutz et al. 2003).

Die Daten der Volkszählung 2001 zeigen, dass die niedrige Fertilität in Wien großteils durch hohe Kinderlosigkeit zustande kam, welche die Kinderlosigkeit in ganz Österreich bei den 19201960 geborenen Frauen um sechs bis neun Prozentpunkte überstieg (Graphik 1, siehe auch Prskawetz et al. 2008 und Spielauer 2005). Mehr als ein Viertel der Wiener Frauen des Geburtsjahrgangs 1960 blieben dauerhaft kinderlos, verglichen mit 16\% aller in Österreich lebenden Frauen. Zusätzlich haben die Mütter in Wien im Mittel weniger Kinder: Die durchschnittliche Anzahl der Kinder pro Mutter bewegte sich bei den seit 1940 geborenen Kohorten um 1,9. In diesem Indikator kann eine allmähliche Konvergenz zum Niveau für Österreich als Ganzes bei den nach 1935 geborenen Kohorten beobachtet werden, d.h. bei denen, die Kinder seit den fünfziger Jahren geboren haben.

\footnotetext{
${ }^{2}$ Die für den Bevölkerungsersatz benötigte TFR beträgt gegenwärtig rund 2.08, lag aber in den 1950er Jahren wegen der höheren Säuglings- und Kindersterblichkeit bei 2.2 (Council of Europe, 1990).
} 


\section{Graphik 1}

Prozentanteil der kinderlos bleibenden Frauen und durchschnittliche Anzahl Kinder pro Mutter in Österreich und in Wien, Geburtskohorten 1920-1960
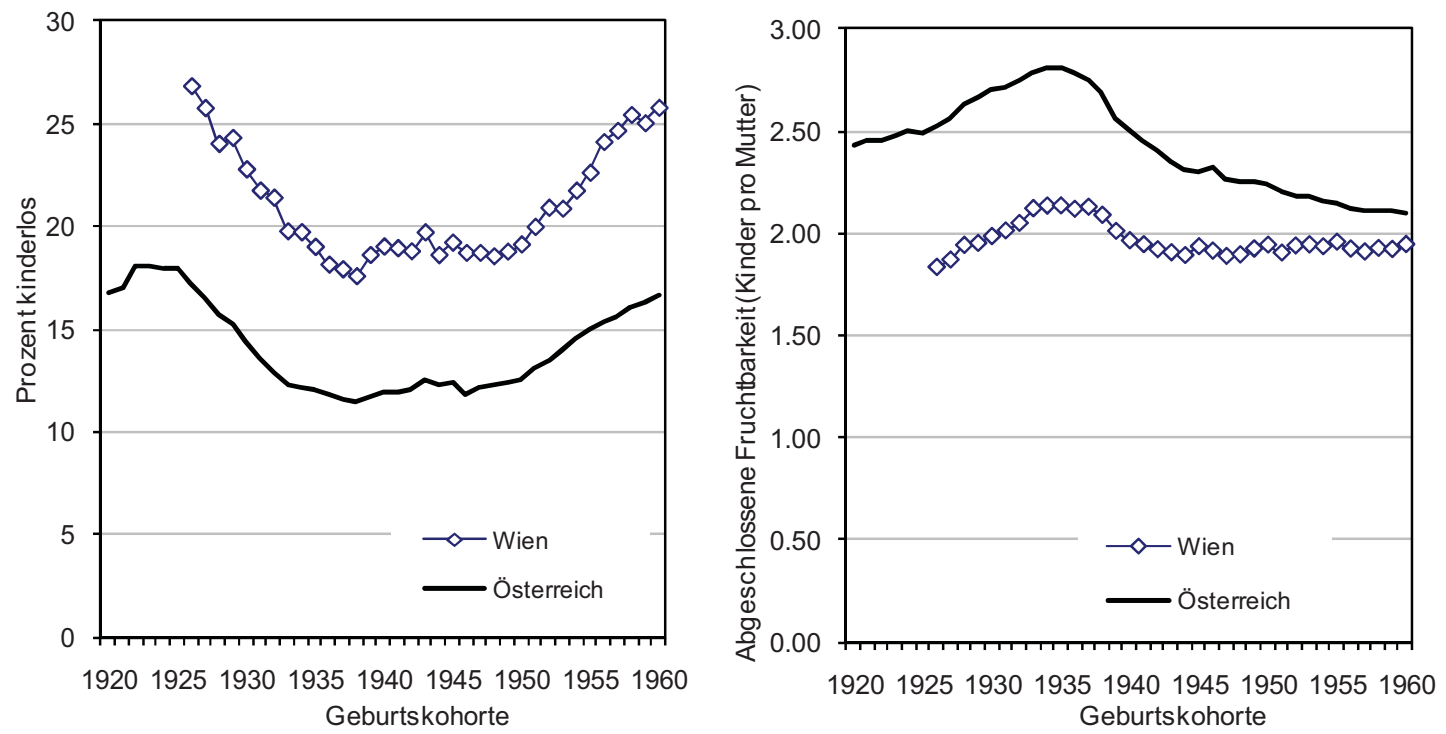

Quelle: Volkszählung 2001 (Statistik Austria 2005)

Für das Wien der letzten 50 Jahre sind einige spezielle Fertilitäts- und Bevölkerungstrends zu beobachten. Die Lücke zwischen den Perioden-Gesamtfruchtbarkeitsziffern Österreichs und Wiens hat sich im Lauf der Zeit geschlossen: Sie fiel von 1,0 im Jahr 1961 auf 0,3 1980 und verschwand in den frühen 2000ern vollständig. Ein Gutteil dieser Konvergenz war dem Rückgang der TFR Österreichs zuzuschreiben, während ein kleiner Aufwärtstrend in der TFR Wiens um 2000 ebenfalls dazu beitrug, den Abstand zu schließen (Graphik 2). In Wien war die TFR in der Nachkriegszeit sehr niedrig, 1951 geschätzt auf 1,11 (Gisser et al. 1975), und auch der Babyboom, der seine Spitze 1963 erreichte, brachte sie nicht über die Schwelle von 1,9. Ein weiteres Tief von 1,24 erreichte sie danach bereits 1977, viel früher als in Gesamtösterreich, wo die niedrigste TFR von 1,33 erst im Jahr 1995 verzeichnet wurde. 


\section{Graphik 2}

Perioden-Gesamtfruchtbarkeitsziffer in Österreich (1951-2009) und Wien (1951, 1961-2009)

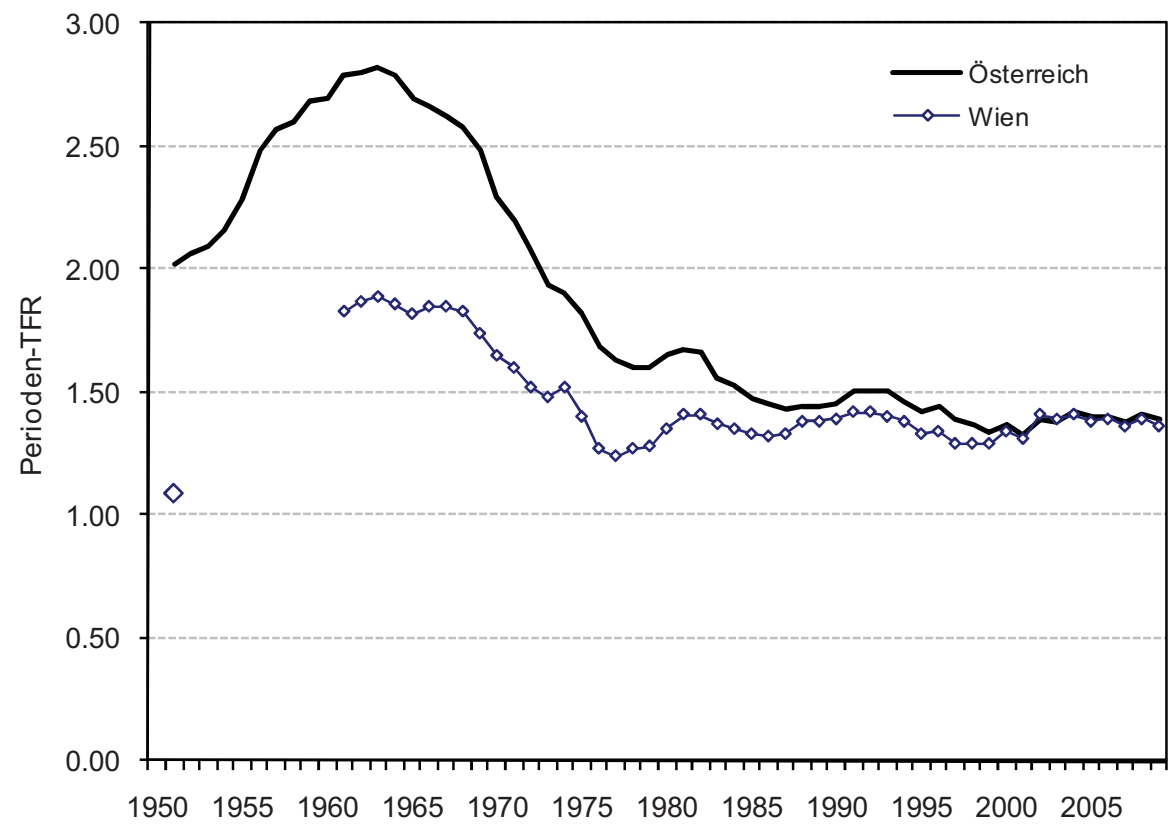

Quelle: VID (2010), Eurostat (2009) und Statistik Austria (2009a) für Österreich; Statistik Austria (2009a) und Gisser et al. (1975, 104, Tabelle 39) für Wien.

Ein bemerkenswerter Umschwung fand auch in der natürlichen Bevölkerungsbilanz (Geburtenbilanz) statt. Viele Dekaden hindurch hatte die Anzahl der Sterbefälle die Anzahl der Lebendgeborenen in Wien mit einer hohen Marge übertroffen, und der Kontrast zu Österreich war ausgeprägt (Graphik 3a). Diese natürliche Bevölkerungsabnahme erreichte in der Mitte der 1970er Jahre ihren Höhepunkt: 1976 waren die Sterbefälle der Wiener Bevölkerung (26.700) doppelt so zahlreich wie die Lebendgeborenen (13.400). Ohne Immigration würde die Wiener Bevölkerung schnell schrumpfen, und tatsächlich schrumpfte sie zwischen Mitte der 1970er und Mitte der 1980er Jahre um fast ein Prozent pro Jahr. Seit Ende der Siebziger verringerte sich der anfänglich hohe Sterbefallüberschuss allmählich und wendete sich nach 2000 in einen Geburtenüberschuss. Nach 2005 übertraf die Rate der natürlichen Zunahme Wiens zum ersten Mal seit dem frühen 20. Jahrhundert diejenige von Gesamtösterreich. Diese bemerkenswerte Wende fand Seite an Seite mit einem neuerlichen Bevölkerungszuwachs seit Ende der 1980er Jahre statt, resultierend aus einer Kombination von steigender Lebenserwartung, wachsender Zuwanderung von Menschen im reproduktiven Alter aus dem Ausland sowie einer geringfügigen Zunahme der Fruchtbarkeitsziffern. Während der erste Faktor hauptsächlich für eine ununterbrochene Verringerung der Anzahl an Sterbefällen in Wien um nicht weniger als 43\% zwischen 1971 und 2009 verantwortlich war, trugen die beiden letzteren Faktoren gemeinsam dazu bei, die Geburtenzahl zwischen 1977 und 2009 um ein Drittel anzuheben (Graphik 3b). Gleichzeitig beeinflusste die umfangreiche Immigration auch in zunehmendem Maße die Fertilitätsmuster und -tendenzen in Wien (siehe Abschnitt 8). 


\section{Graphik 3a}

Natürliches Bevölkerungswachstum (pro 1.000 der Bevölkerung) in Österreich und Wien (19602009)

\section{Graphik 3b}

Anzahl der Lebendgeborenen und Sterbefälle in Wien, 1961-2009
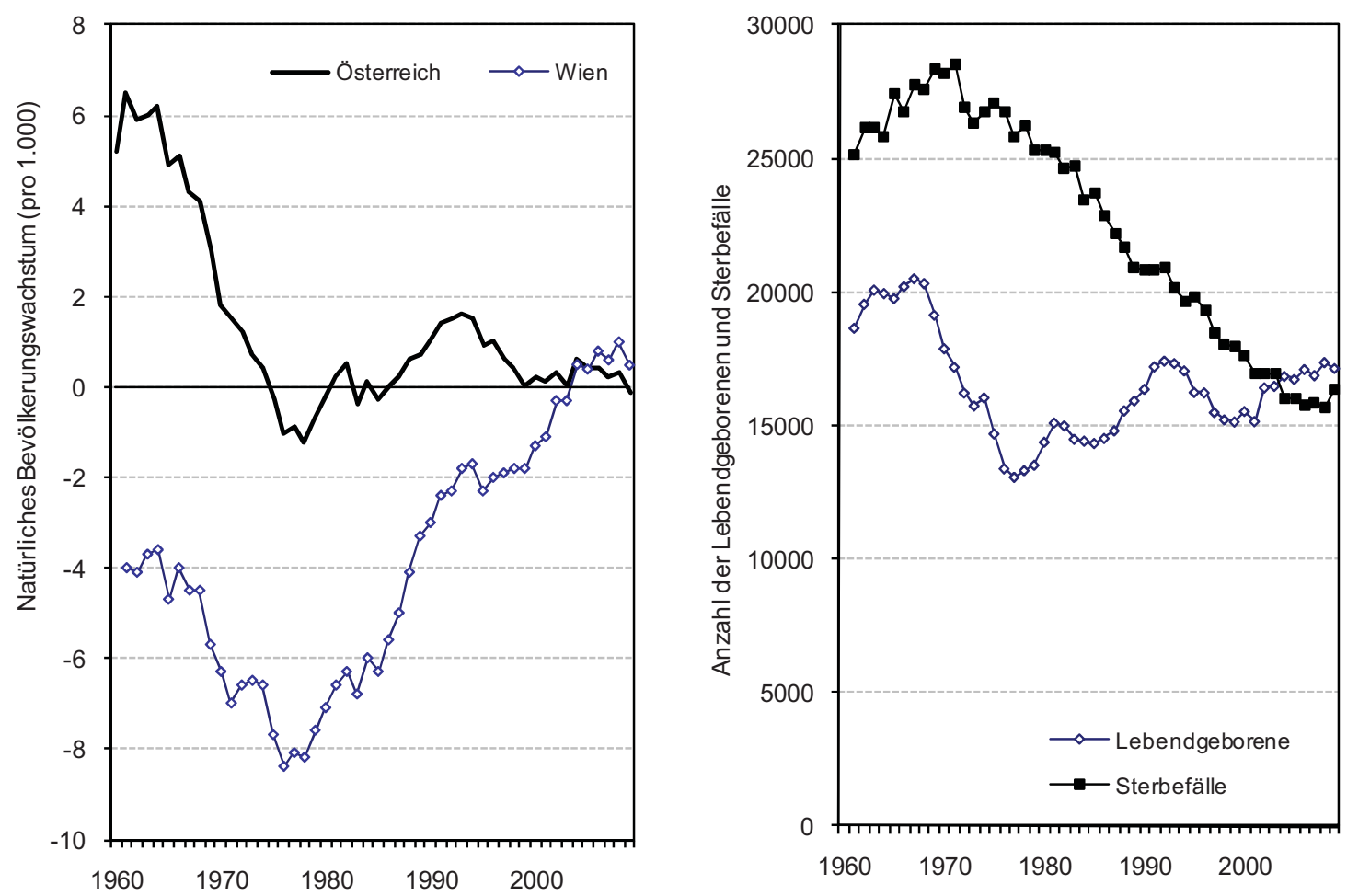

Quelle: Statistik Austria (2010)

Die angesprochenen Unterschiede bezüglich der Fruchtbarkeits- und Bevölkerungsentwicklungen zwischen Wien und den übrigen Bundesländern Österreichs machen die Analyse und das Monitoring der Fertilitätstendenzen in Wien besonders interessant. Die hier analysierten Daten erlauben es auch, den Einfluss der Zuwanderung vom Ausland auf die Gestaltung der Geburtentrends zu untersuchen. Um die spezielle Position Wiens in den österreichischen Bevölkerungsentwicklungen hervorzuheben, werden die Resultate für Wien häufig mit den kombinierten Daten für die anderen Bundesländer Österreichs und mit dem ganzen Land kontrastiert. Der folgende Abschnitt fasst die Hauptziele des Geburtenbarometers Wien sowie die Forschungsfragen zusammen, die mit diesen neuen Fertilitätsdaten angesprochen werden können. Anschließend werden die verwendeten Daten und die Methodologie sowie die in dieser Studie analysierten Indikatoren umrissen. Dann wird ein ausführlicher Überblick über die jährlichen Fertilitätsindikatoren gegeben, einschließlich einer Betrachtung der Altersmuster der Fertilität und insbesondere jener bei Erstgeburten sowie einer kurzen Analyse der Rolle von Tempoeffekten auf die im Geburtenbarometer benutzten Periodenfertilitätsindikatoren. Es folgt ein Blick auf die Tendenzen in den vierteljährlichen Wiener Fruchtbarkeitsziffern. Zusätzlich wird der Einfluss der im Ausland geborenen Frauen auf die beobachteten Fertilitätsentwicklungen in Wien seit 1999 analysiert. Das Schlusskapitel fasst die Hauptergebnisse in einer breiteren Perspektive der Wiener Bevölkerungsdynamik zusammen.

\section{Hauptziele und Probleme}

Analog zum Geburtenbarometer Österreich zielt das Geburtenbarometer Wien hauptsächlich auf ein aktuelles Fertilitätsmonitoring in Wien ab, basierend auf einem Satz jährlicher und vierteljährlicher 
Fruchtbarkeitsziffern und -indikatoren und regelmäßig erscheinenden summarischen Berichten. Im Unterschied zum Geburtenbarometer Österreich, das Monatsreihen von Fertilitätsdaten zur Verfügung stellt, enthält das Wiener Geburtenbarometer nur vierteljährliche Zeitreihen, um zufällige Schwankungen in den Geburtenzahlen zu verringern, die in kleineren, subnationalen Bevölkerungen stärker sind. Die Quartalsdaten werden sowohl saisonal (regelmäßige Saisonschwankungen in den Geburtenziffern) als auch kalendarisch (Anzahl der Kalendertage in jedem Quartal) bereinigt. Die Berichte für Wien vergleichen Fruchtbarkeitsentwicklungen mit jenen im gesamten Bundesgebiet und heben somit wichtige Unterschiede bzw. gegenläufige Trends hervor. ${ }^{3}$ Das vierteljährliche Datenformat ermöglicht einen Vergleich sozialer, ökonomischer und politischer Tendenzen mit Fertilitätstrends und erlaubt Fragestellungen wie „Hat die Wirtschaftskrise die Erstgeburtenziffern beeinflusst?“ oder „Korrelieren Verbesserungen in der Kinderbetreuung mit Änderungen in den Fruchtbarkeitsziffern?" Das Geburtenbarometer Wien zielt auch darauf ab, vergleichende Forschung und Analysen der Wiener Fertilität zu erleichtern. Wien mit seiner verschiedenartigen Bevölkerung und der starken Immigration kann als Beispiel einer bedeutenden europäischen Stadt mit eigenartigen Fruchtbarkeitstendenzen gesehen werden. Innerhalb dieser Perspektive ergeben sich einige interessante Forschungsfragen:

- Was sind die Gründe für die Konvergenz der Perioden-Fertilitätsraten zwischen Wien und Gesamt-Österreich?

- Hat eine ähnliche Konvergenz in den Erstgeburtenziffern und in den Übergangsraten zu den Geburten zweiter, dritter und höherer Ordnung stattgefunden?

- Bleibt die Kinderlosigkeitsquote in Wien deutlich über der für ganz Österreich?

- Hat die steigende Anzahl im Ausland geborener Mütter zur geringen Zunahme der Perioden-Fertilitätsrate in Wien nach 1977 und zu ihrer Konvergenz mit dem übrigen Österreich beigetragen?

- Verschwinden die Unterschiede in den Fruchtbarkeitsziffern zwischen den im Ausland und den in Österreich geborenen Frauen in Wien?

Diese Fragen sind direkt mit der Datenbank für das Geburtenbarometer Wien bearbeitbar und werden hier angesprochen. Zukünftige Erweiterungen dieser Analysen könnten weitere Dimensionen hinzufügen, insbesondere Bildung und Religion. ${ }^{4}$ Wien hat die am höchsten gebildete, religiös verschiedenartigste und auch die ,weltlichste“ Bevölkerung in Österreich. Diese Faktoren haben die Wiener Fertilitätstendenzen in der Vergangenheit geformt und zu der beständig niedrigen Geburtenhäufigkeit in Wien im letzten Jahrhundert beigetragen.

\section{Daten}

Das Geburtenbarometer erfordert ausführliche Daten über Lebendgeburten, spezifiziert nach dem Bundesland des Wohnsitzes des Mutter, dem Alter der Mutter, der Geburtenfolge, dem zeitlichen Abstand seit der letzten vorangegangenen Geburt (für Mütter zweiter und folgender Geburten) sowie dem Geburtsland der Mutter (hier wird nur zwischen in Österreich und außerhalb Österreichs geborenen Müttern unterschieden, weil ausführlichere Daten erst seit 2008 verfügbar sind). Die Daten, die alle diese Merkmale enthalten, sind seit dem Jahr 1984 vorhanden, welches auch das Startjahr einiger der Datenreihen des Geburtenbarometers ist.

\footnotetext{
${ }^{3}$ Der erste reguläre Bericht des Wiener Geburtenbarometers bezieht sich auf das Jahr 2009 und wurde gemeinsam mit dem Bericht über das gesamtösterreichische Geburtenbarometer publiziert, vgl.

http://www.oeaw.ac.at/vid/download/Geburtenbarometer_Ergebnis_Jahr2009.pdf

${ }^{4}$ Fertilitätsdifferentiale in der Wiener Bevölkerung nach Religion werden 2011-2014 am Vienna Institute of Demography in dem Projekt Past, present and future religious prospects in Vienna 1950 - 2050 unter der Leitung von Anne Goujon untersucht.
} 
Die Statistik Austria hat für das Projekt Auszüge aus den Geburtendatensätzen 1984-2010 zur Verfügung gestellt, welche die Konstruktion aller dargestellten Indikatoren der Periodenfruchtbarkeit erlauben. Es handelt sich um Daten über alle lebend geborenen Kinder in Wien und aus Vergleichsgründen in Österreich zwischen Jänner 1984 und September 2010; diese umfassen 433.000 Datensätze für Wien und 2,2 Millionen für Österreich. Diese Datensätze werden auch verwendet, um die „Risikopopulation“ für die paritätsspezifischen Familienzuwachswahrscheinlichkeiten zu schätzen, nämlich die Anzahl der Frauen nach der Parität und dem Jahr, in dem sie eine gegebene Parität erreichten.

Die Schätzung des Nenners (weibliche „Risikobevölkerung“) erforderte die Kombination verschiedener Datenquellen. Für den allgemeinsten und meistbenutzten Periodenfertilitätsindikator, die Perioden-Gesamtfruchtbarkeitsziffer (Total Fertility Rate, TFR), wurde die Altersgliederung der 12- bis 55-jährigen Frauen nach einzelnen Lebensjahren im Zeitraum 1984-2009 verwendet, zur Verfügung gestellt von der Statistik Austria (ISIS-Datenbank) und vom Referat für Statistik und Analyse (MA 5) der Stadt Wien. Für 2002-2009 wurde auch die Altersgliederung der weiblichen Bevölkerung Wiens und Österreichs nach dem Geburtsland (unterschieden nur nach Österreich und allen anderen Ländern zusammen) herangezogen. Für die Berechnung des paritäts- und alterskontrollierten Index der Fertilität (PATFR) 2002-2009, der zur Analyse der Erstgeburtstrends dient, wurden die Volkszählungsdaten 2001 bezüglich der Alters- und Paritätsverteilung der in Wien wohnhaften Frauen verwendet und mit eigenen vierteljährlichen und jährlichen Schätzungen der alters- und ordnungsspezifischen Fruchtbarkeitsziffern 2001-2010 aktualisiert. Zusätzliche Zeitreihen der Verteilung der weiblichen Bevölkerung nach Alter und Parität in den Jahren 1991-2001 wurden an Hand der Volkszählungsdaten 1991 geschätzt. Um schließlich die jüngsten vierteljährlichen Daten zu berechnen, wurde die Zahl der Wienerinnen nach dem Alter am Beginn jedes Quartals 2010 mit Hilfe der für 1. Jänner 2010 publizierten Daten und der altersspezifischen relativen vierteljährlichen Bevölkerungsveränderung während des Jahres 2009 geschätzt.

\section{Methoden und Indikatoren}

Die im Geburtenbarometer Wien verwendete Methodologie ist größtenteils identisch mit jener, die 2005 für das österreichische Geburtenbarometer erstellt und im Detail im Artikel von Sobotka et al. (2005) beschrieben wurde, insbesondere in seinen Online-Anhängen. ${ }^{5}$ Hier werden einige wenige Unterschiede umrissen und alle benutzten Indikatoren spezifiziert.

Die Berechnung von Fertilitätsraten für Zeiträume, die kürzer sind als ein Kalenderjahr, erfordert die Durchführung von Korrekturen in Bezug auf Saisonalität sowie von Kalenderadjustierungen (in Bezug auf die unterschiedliche Zahl von Tagen in einem Monat oder in einem Quartal und in Bezug auf die Zahl der Wochentage, wenn die Geburtenzahlen nach Wochentagen schwanken). Im Geburtenbarometer für Österreich wird für diese Bereinigungen die X-12-ARIMAMethode des Softwarepakets Gretl (Cottrell 2004) angewendet (Sobotka et al. 2005), und die Saisonkorrektur wird separat für die Geburten der Ordnungen 1, 2 und 3+ berechnet. Das gleiche Verfahren wird für die Bereinigung der vierteljährlichen Wiener Daten verwendet, mit einer Vereinfachung: Die Daten werden nicht nach der Anzahl der Wochentage in jedem Vierteljahr korrigiert, da die vierteljährlichen Geburtenzahlen dadurch nur in sehr geringem Umfang beeinflusst werden.

\section{Fertilitätsindikatoren im Geburtenbarometer Wien}

\footnotetext{
${ }^{5}$ Verfügbar auf http://www.oeaw.ac.at/vid/download/Sobotka_et_al_Appendices_031005.pdf
} 
In Einklang mit dem Geburtenbarometer für Österreich werden zwei Hauptindikatoren der Perioden-Gesamtfruchtbarkeit berechnet: die herkömmliche Perioden-Gesamtfertilitätsrate (Total Fertility Rate, TFR) und die Durchschnittliche Perioden-Parität (Period Average Parity, PAP). Wie in der demographischen Analyse üblich, werden alle Indikatoren von den Daten über Lebendgeborene berechnet und Totgeborene nicht berücksichtigt.

\section{Die Gesamtfruchtbarkeitsziffer (TFR)}

Dieser Indikator wird als Summe der altersspezifischen Fruchtbarkeitsziffern, f(x), für einen gegebenen Zeitraum t berechnet (Kalenderjahr oder Vierteljahr), wobei die Frauen des gesamten reproduktiven Alters einbezogen werden (Alter 12 bis 55 Jahre im Geburtenbarometer Wien):

$$
\operatorname{TFR}(t)=\sum_{x=12}^{55} f(x, t), \text { wobei } f(x, t)=B(x, t) / P^{F}(x, t) .
$$

Dabei stellt $\mathrm{B}(\mathrm{x})$ die Anzahl der Lebendgeborenen von Frauen des Alters $\mathrm{x}$ dar. $\mathrm{P}^{\mathrm{F}}(\mathrm{x})$ steht für die durchschnittliche Anzahl der Frauen des Alters $x$ in der Bevölkerung im Zeitraum t, geschätzt als einfacher Durchschnitt zwischen den Zahlen der Frauen am Beginn und am Ende eines Jahres oder Vierteljahres. Die TFR wird seit 1984 für jedes Kalenderjahr und seit dem ersten Quartal 2002 zusätzlich für jedes Vierteljahr berechnet. Für die Zeit seit 2002 werden die jährlichen TFR-Reihen auch für in Österreich geborene und für außerhalb Österreichs geborene Frauen separat berechnet. Bei der Berechnung vierteljährlicher Daten wird die TFR weiters mit dem Faktor vier sowie mit einem Index, der Saison- und Kalendereffekte bereinigt, multipliziert.

\section{Der paritäts- und alterskontrollierte Fertilitätsindex für Erstgeburten (PATFR $)_{1}$}

Dies ist ein Index der Erstgeburtenziffer, der zusätzlich zur Altersdimension, wie sie zur Konstruktion der Perioden-TFR verwendet wird, auch die Paritätsverteilung der weiblichen Bevölkerung berücksichtigt. Im Geburtenbarometer wird der $\mathrm{PATFR}_{1}$ von den altersspezifischen Wahrscheinlichkeiten $\left(\mathrm{q}_{1}(\mathrm{x})\right)$, ein erstes Kind im Alter $\mathrm{x}$ während eines gegebenen Zeitraums $\mathrm{t}$ zu gebären, spezifiziert für die kinderlosen Frauen, berechnet:

$$
\mathrm{q}_{1}(\mathrm{x}, \mathrm{t})=\mathrm{B}_{1}(\mathrm{x}, \mathrm{t}) / \mathrm{P}_{0}^{\mathrm{F}}(\mathrm{x}, \mathrm{T}),
$$

wobei $\mathrm{P}_{0}^{\mathrm{F}}(\mathrm{x}, \mathrm{T})$ die Gesamtzahl kinderloser Frauen im Alter x zu Beginn des Zeitraums t bezeichnet. ${ }^{6}$ Für vierteljährliche Berechnungen werden Kalender- und Saisonbereinigung für jedes einzelne Alter separat durchgeführt, und jede altersspezifische Erstgeburtswahrscheinlichkeit $\mathrm{q}_{1}(\mathrm{x}, \mathrm{t})$ wird mit 4 multipliziert. Der Gesamtfertilitätsindex für erste Geburten (PATFR 1 ) wird wie folgt berechnet:

$$
\operatorname{PATFR}_{1}(t)=1-\prod_{x=12}^{55}\left[1-q_{1}(x, t)\right] .
$$

Diese Berechnungsweise des Perioden-PATFR folgt der Methodologie und den Notationen, die von Rallu und Toulemon (1994) eingeführt wurden. ${ }^{7}$ Alter x bezieht sich auf das während des

\footnotetext{
${ }^{6}$ Es wurden die Frauen berücksichtigt, die in einem gegebenen Kalenderjahr die Alter 12 bis 55 Jahre erreichten. Einige wenige Geburten, die Frauen vor dem 12. oder nach dem 55. Lebensjahr hatten, wurden den 12- bzw. 55-jährigen Frauen zugeschlagen.

${ }^{7}$ Eine alternative Methode der Berechnung des paritäts- und altersspezifischen Fertilitätsindex PATFR aus paritäts- und altersspezifischen Fertilitätsraten $m_{i}(x)$ wird in der vor kurzem errichteten Human Fertility Database (www.humanfertility.org) verwendet und im Detail von Jasilioniene et al. (2010) beschrieben.
} 
Kalenderjahres erreichte Alter, das als Differenz zwischen dem Berichtsjahr und dem Geburtsjahr der Frau (Mutter) berechnet wird. Da die Schätzung der Risikobevölkerung von kinderlosen Frauen nach dem Alter auf Basis der Volkszählung 2001 erfolgte, wurden vierteljährliche und jährliche Zeitreihen des $\mathrm{PATFR}_{1}$ für Wien für die Zeit seit 2002 berechnet. Zusätzlich wurde die jährliche Reihe des PATFR $_{1}$ auch für den Zeitraum 1991-2001 unter Verwendung der Expositionsdaten der Bevölkerung berechnet, die auf der Volkszählung 1991 basierten.

\section{Familienzuwachswahrscheinlichkeiten nach Dauer seit der letztvorherigen Geburt $\left(P P R_{i, i+1}\right)^{8}$}

In den Familienzuwachswahrscheinlichkeiten („Paritätsprogressionsraten“ PPR), wie sie in diesem Projekt verwendet werden, ist die Übergangsrate zwischen den verschiedenen Paritäten eine Funktion der seit der jeweils letzten Geburt vergangenen Zeit. Im Gegensatz zum oben spezifizierten PATFR-Index wird die Zeitdauer seit der letzten vorangegangenen Geburt anstatt des Alters als Hauptparameter des Fertilitätsverhaltens von Frauen, die mindestens ein Kind haben, angesehen. Ein zusammenfassender Indikator, der die Fruchtbarkeitsziffern über alle betrachteten Geburtenabstände kombiniert, ergibt die Perioden-Familienzuwachswahrscheinlichkeit ( $\left.P_{P R}, i+1\right)$. Dabei bezeichnet die Notation i, $i+1$ einen Übergang von Parität i zur folgenden Parität (i+1); so bezeichnet man etwa die Familienzuwachswahrscheinlichkeit vom zweiten zum dritten Kind als $\mathrm{PPR}_{2,3}$.

Das Geburtenbarometer verwendet Familienzuwachswahrscheinlichkeiten, die aus dem durch die Dauer (in Jahren) seit der letzten vorangegangenen Geburt spezifizierten Satz der Geburtenfolgewahrscheinlichkeiten gebildet werden. Dieser Ansatz erfordert eine Schätzung der Verteilung der weiblichen Bevölkerung nach der Parität und dem Jahr der vorigen Geburt für jeden betrachteten Zeitraum. ${ }^{9}$ Dauer- und paritätsspezifische Geburtenfolgewahrscheinlichkeiten werden für Geburten zweiter und höherer Ordnung berechnet. Die Wahrscheinlichkeit für eine Frau, die ihren gegenwärtigen Paritätsstatus (i) im Jahr y = t-d erreicht hat, während des Jahres $t$ ein weiteres Kind zu gebären, wird wie folgt berechnet:

$$
q_{i+1, d}(t)=B_{i+1, d}(t) / P_{i, d}^{F}(t)
$$

wobei $P_{i, d}^{F}(t)$ die Anzahl der Frauen ist, die im Jahr y = t-d ein Kind der Ordnung i bekamen und am Beginn des Jahres $\mathrm{t}$ weiterhin in Parität $\mathrm{i}$ blieben, und $B_{i+1} \mathrm{~d}(t)$ die Anzahl der Geburten der Ordnung i+1 während des Jahres t bei Frauen, die ihre letzte vorhergehende Geburt im Jahr t-d hatten. In dieser Berechnung stellt d die Dauer des Verweilens in einer gegebenen Parität dar, geschätzt als d = t-y ( $\mathrm{y}$ ist das Jahr der letzten vorangegangenen Entbindung). Es wurden Expositionen für alle Dauern bis 25 Jahre geschätzt. Die höchste betrachtete Geburtenfolge betrifft einen Familienzuwachs mit offenem Ende zur Geburt fünfter oder höherer Ordnung bei Frauen, die vier oder mehr Kinder hatten:

$$
q_{5+, d}(t)=B_{5+, d}(t) / P_{4+, d}^{F}(t)
$$

\footnotetext{
${ }^{8}$ Eine kurze Beschreibung dauer- und paritätsspezifischer Indikatoren findet sich bei Rallu und Toulemon (1994); mehr Details über die beim Geburtenbarometer für Österreich verwendete Methodologie enthalten die Appendices 2 und 3 bei Sobotka et al. (2005).

${ }^{9}$ Detaillierte Zeitreihen von Geburtendaten beginnen in Österreich erst 1984. Um die Anzahl der Mütter nach Parität und dem Jahr der letztvorangegangenen Geburt zu schätzen, wurde darauf abgezielt, eine genügend lange „Expositionsperiode“ zu haben, in diesem Fall mindestens 15 Jahre (1984-1998). Daher wurden alle dauer- und paritätsspezifischen Geburtenfolgewahrscheinlichkeiten sowie Familienzuwachswahrscheinlichkeiten nur von 1999 an berechnet und für die früheren Jahre die „Risikobevölkerung“ der Frauen mit langen Dauern seit der vorherigen Geburt, d.h. mit Geburten vor 1984, geschätzt. Da sehr wenige Frauen ein weiteres Kind nach Intervallen von mehr als 15 Jahren bekommen, hatten diese Schätzungen der „Risikopopulation“ mit langer Dauer keinen Einfluss auf die errechneten Familienzuwachswahrscheinlichkeiten.
} 
Für vierteljährliche Daten wird die Kalender- und Saisonbereinigung für jede dauer- und paritäts-spezifische Wahrscheinlichkeit separat durchgeführt, und jede Wahrscheinlichkeit wird mit vier multipliziert. Die Risikobevölkerung wird für den Anfang jedes Vierteljahres geschätzt.

Familienzuwachswahrscheinlichkeiten (PPR) wurden für Frauen der Paritäten 1, 2 und 3 und die offene Paritätskategorie 4+ berechnet:

$$
P P R_{i, i+1}(t)=1-\prod_{d=0}^{25}\left[1-q_{i+1, d}(t)\right]
$$

Für die höchste berücksichtigte Geburtenordnung gibt die Familienzuwachsberechnung die kumulative Übergangsrate zu Geburten fünfter und höherer Ordnung bei Frauen mit vier oder mehr Kindern wieder, und die Berechnung kann wie folgt vereinfacht werden:

$$
\operatorname{PPR}_{4+, 5+}(t)=\sum_{d=0}^{25} q_{5+, d}(t) .
$$

In dieser Berechnung werden Mehrlingsgeburten als Geburten verschiedener Ordnung behandelt. Die geschätzte Bevölkerung der Frauen nach Parität und Jahr der letzten vorangegangenen Geburt berücksichtigt nicht die Einflüsse der Sterblichkeit und der Migration, wobei letztere in Wien besonders intensiv ist. Daher ist die „Null-Migrations-Annahme“ problematisch; die tatsächlichen Zahlen der Frauen nach Parität und Dauer seit der vorhergehenden Geburt sind von der Immigration betroffen und weichen insofern von den vorliegenden Schätzungen für das Projekt ab. Da keine Daten über die Paritäts- und Geburtenabstands-Verteilung von Immigrantinnen vorliegen, wurde entschieden, diesen Effekt zu ignorieren. Auf Grund vorliegender Forschungen ist zu erwarten, dass das eingesetzte Modell dennoch stabile Resultate liefert, die vermutlich zu einer geringfügigen Überschätzung der ,wirklichen“ Familienzuwachswahrscheinlichkeiten in der Größenordnung einiger Prozentpunkte führen. ${ }^{10}$ Es ist geplant, dieser Frage im Rahmen zukünftiger Forschungen nachzugehen.

\section{Durchschnittliche Perioden-Parität (PAP)}

Die Durchschnittliche Perioden-Parität (PAP) wird für jede Paritätskategorie $j>1$ berechnet durch die Kombination des PATFR-Index für Parität 1 mit den Familienzuwachswahrscheinlichkeiten für die Paritäten bis j:

$$
\operatorname{PAP}_{j}(t)=\operatorname{PATFR}_{l}(t) \prod_{i=1}^{j-1} P P R_{i, i+1}(t)=P A P_{j-1}(t) \cdot P P R_{j-1, j}(t)
$$

Die höchste Familienzuwachswahrscheinlichkeits-Kategorie (4+ zu 5+) betrifft die Progression von der vierten Geburt zur fünften und allen folgenden Geburten:

$$
\operatorname{PAP}_{5+}(t)=P A T F R_{1}(t) \prod_{i=1}^{4+} P P R_{i, i+1}(t)=P A P_{4} \cdot P P R_{4+, 5+}(t)
$$

Der Gesamtindex der durchschnittlichen Periodenparität wird wie folgt berechnet:

\footnotetext{
${ }^{10}$ Während der Zähler (die Geburtenzahl) in den vorliegenden Berechnungen vollständig ist, wird der Nenner - die Anzahl der Frauen nach aktueller Parität und Jahr der Geburt des letzten vorangegangenen Kindes - unterschätzt, woraus sich höhere Werte für die abgeleiteten Fertilitätsindikatoren ergeben.
} 


$$
P A P(t)=\operatorname{PATFR}_{l}(t)+\sum_{\mathrm{j}=2}^{5+} P A P_{j}(t)
$$

Die Durchschnittliche Perioden-Parität PAP wird für die gesamte Periode berechnet, für die sowohl PATFR 1 und $\mathrm{PPR}_{\mathrm{i}, \mathrm{i}+1}$ erstellt werden, nämlich für 1999-2009.

Tabelle 1 verzeichnet zusammenfassend die für das Geburtenbarometer Wien berechneten Indikatoren sowie die Zeiträume, für die sie berechnet werden. Der folgende Abschnitt gibt einen Überblick über die Hauptresultate und vergleicht die berechneten Indikatoren für die Stadt Wien mit Österreich als Ganzem und/oder dem übrigen Bundesgebiet (Österreich ohne Wien).

\section{Tabelle 1}

Indikatoren für das Geburtenbarometer Wien

\begin{tabular}{|l|l|l|l|}
\hline & $\begin{array}{c}\text { Jährliche } \\
\text { Daten }\end{array}$ & $\begin{array}{l}\text { Vierteljährli- } \\
\text { che Daten }\end{array}$ & \multicolumn{1}{|c|}{ Bemerkungen } \\
\hline $\boldsymbol{T F R}$ & $1984-2009$ & $\begin{array}{l}2002-2010 \\
(\mathrm{Q} 3)\end{array}$ & $\begin{array}{l}\text { Die TFR wird auch nach der Geburtenfolge berechnet (TFRi). } \\
\text { Zusätzlich wurde die jährliche TFR-Zeitreihe 2002-2009 für in } \\
\text { Österreich sowie im Ausland geborene Frauen berechnet. }\end{array}$ \\
\hline $\boldsymbol{P A T F R}_{\boldsymbol{1}}$ & $1991-2009$ & $\begin{array}{l}2002-2010 \\
(\mathrm{Q} 3)\end{array}$ & $\begin{array}{l}\text { Bruch in der Zeitreihe 2002 (neue Risikopopulation basierend } \\
\text { auf der Volkszählung 2001). }\end{array}$ \\
\hline $\boldsymbol{P P R}_{\boldsymbol{i}, \boldsymbol{i}+\boldsymbol{1}}$ & $1999-2009$ & $\begin{array}{l}2002-2010 \\
(\mathrm{Q} 3)\end{array}$ & \\
\hline $\boldsymbol{P A P}$ & $1999-2009$ & $\begin{array}{l}2002-2010 \\
(\mathrm{Q} 3)\end{array}$ & Bruch in der Zeitreihe 2002 (neue Reihe der PATFR $_{l}$ ). \\
\hline
\end{tabular}

\section{Hauptergebnisse im Überblick: Jährliche Daten}

Dieser Abschnitt konzentriert sich auf die jährlichen Resultate; ausgewählte Ergebnisse der vierteljährlichen Analyse folgen im nächsten Abschnitt. Zuerst werden die Trends in der PeriodenGesamtfruchtbarkeitsziffer betrachtet. Während in der Mitte der 1990er Jahre die TFR in Wien um mehr als 10\% niedriger war als in den anderen österreichischen Regionen zusammen und sich die absolute Differenz 1984 auf 0,2 belief, verschwand dieser Unterschied praktisch in den frühen 2000er Jahren. Ein Großteil dieser Lücke schloss sich zwischen 1997 und 2002, als die TFR in Wien vom Tief 1,29 wieder auf 1,41 stieg (Graphik 4). 


\section{Graphik 4}

Perioden-Gesamtfertilitätsrate (TFR) in Wien, im übrigen Bundesgebiet und in Österreich als Ganzes (1984-2009)

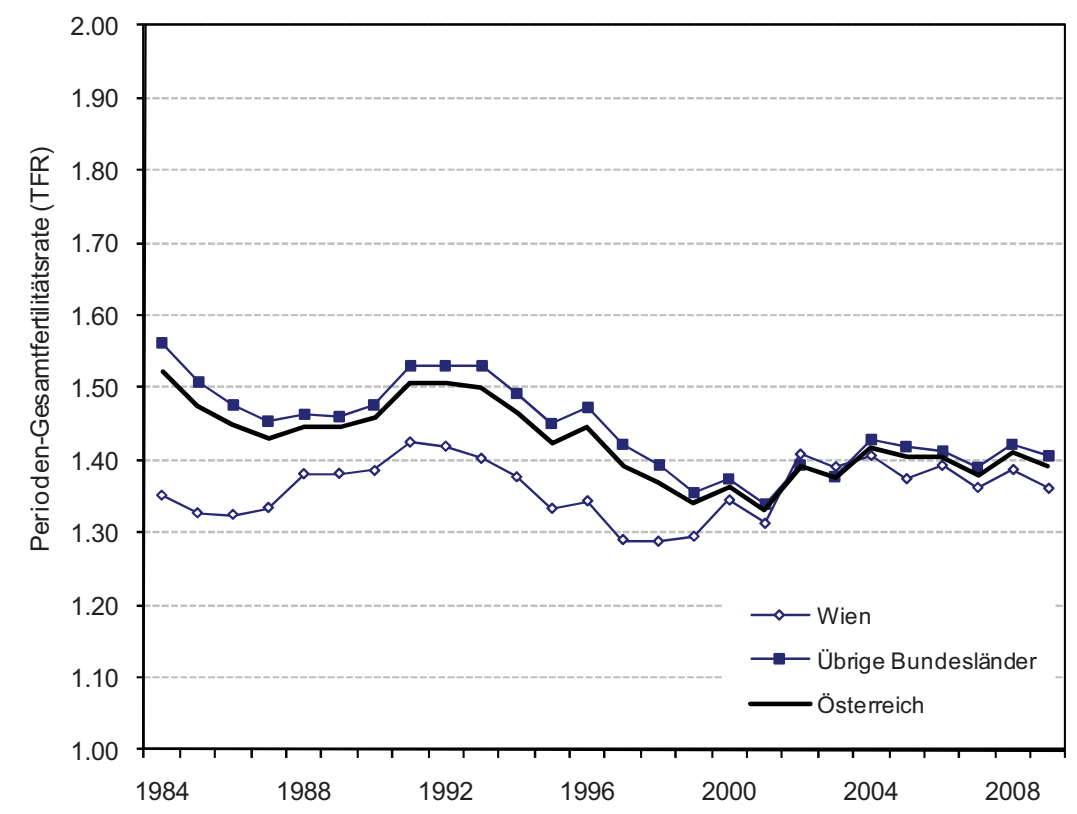

Quelle: Geburtenbarometer Wien, Berechnungen der Autoren

Wird die Perioden-TFR in ihre ordnungsspezifischen Bestandteile zerlegt, so wird offenbar, dass der Hauptfaktor für die Konvergenz in der TFR zwischen Wien und dem übrigen Österreich die Konvergenz in der TFR für dritte und Geburten höherer Ordnung war. Während die TFR für Erstgeburten $\left(\mathrm{TFR}_{1}\right)$ in Wien durchwegs seit 1984 die des übrigen Bundesgebiets übertraf, die für zweite Geburten $\left(\mathrm{TFR}_{2}\right)$ dagegen konsistent um beträchtliche $15-20 \%$ unterhalb des Niveaus der anderen Bundesländer lag, stagnierte die TFR für Geburten dritter und die höherer Ordnung $\left(\mathrm{TFR}_{3+}\right)$ in Wien zuerst und stieg dann kurz an - zu einer Zeit, als sie im übrigen Österreich sank. Durch den kurzen Schwall zwischen 1999 und 2003 schloss sich schließlich die Lücke, die 1984 in absoluter Hinsicht noch 0,16 betragen hatte. Seit 2003 blieb die $\mathrm{TFR}_{3+}$ in Wien und im übrigen Bundesgebiet mit jeweils ungefähr 0,25 stabil (Graphik 5). 


\section{Graphik 5}

Ordnungsspezifische Komponenten der Perioden-TFR in Wien und im übrigen Österreich
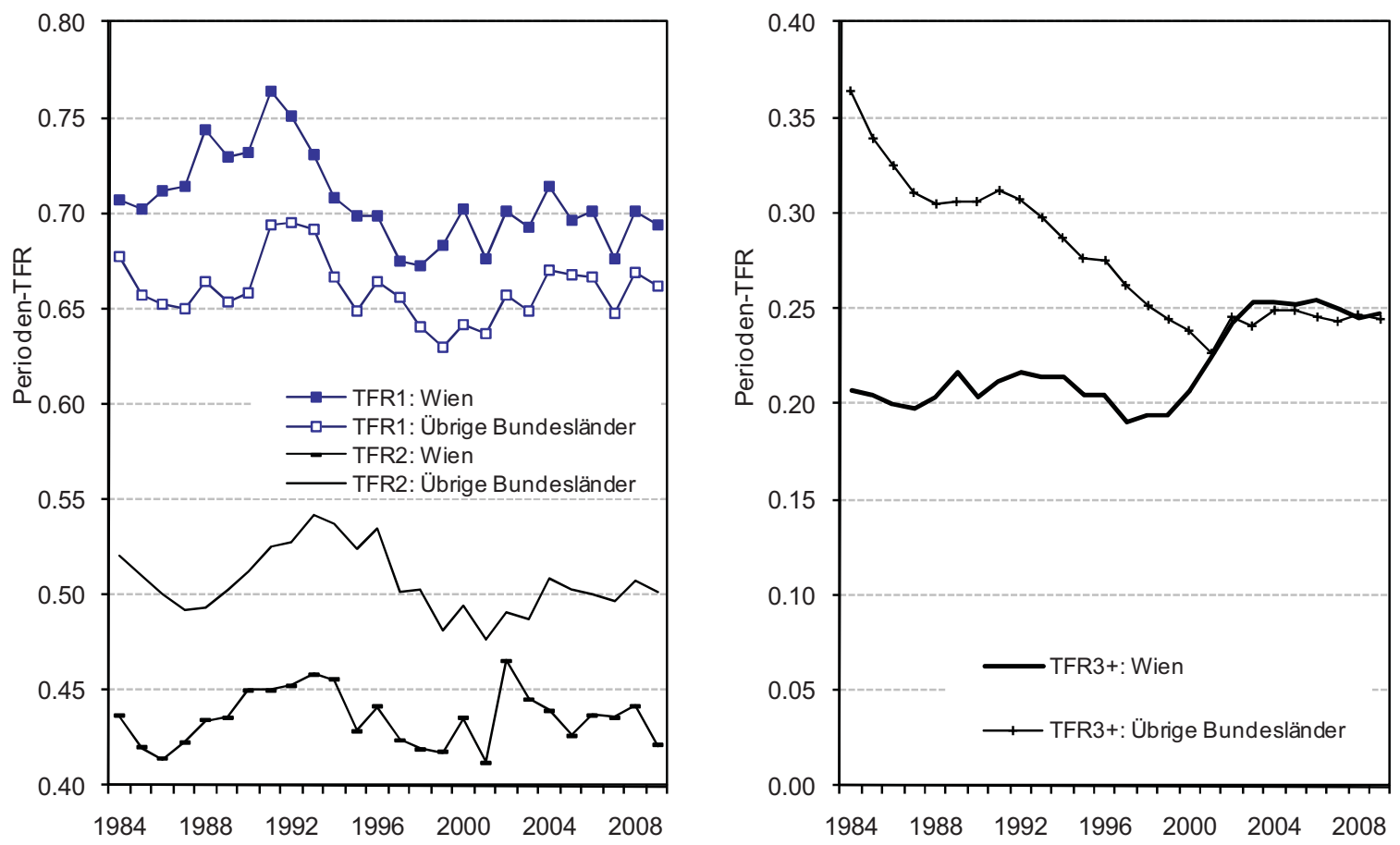

Quelle: Geburtenbarometer Wien, Berechnungen der Autoren

Allerdings kann die Perioden-TFR ein verzerrtes Abbild der Fertilitätstendenzen und -niveaus geben, weil sie die tatsächliche Paritätsverteilung in der weiblichen Bevölkerung nicht berücksichtigt und durch Änderungen im Timing der Geburten stark beeinflusst werden kann. Folglich werden die Trends in der Durchschnittlichen Perioden-Parität (PAP) und in ihren ordnungsspezifischen Bestandteilen näher betrachtet, um zu überprüfen, ob die allgemeine Konvergenz in der Fruchtbarkeit und besonders in den Geburtenziffern höherer Ordnung auch mit diesen geeigneteren Periodenfertilitätsindikatoren bestätigt wird. Wegen der kürzeren Zeitreihen der PAP für Wien richtet sich die Analyse nur auf den Zeitraum zwischen 1999 und 2009. Graphik 6 zeigt, dass die PAP in Wien weiterhin ein wenig unter der PAP in den anderen Regionen Österreichs bleibt, obgleich sich dieser Unterschied in jüngster Zeit absolut auf weniger als 0,1 verringert hat. Der durch die TFR angezeigte Aufschwung in der Fertilität zu Ende der 1990er Jahre und in den frühen 2000ern wird auch durch die PAP bestätigt, die zwischen 1999 und 2004 von 1,40 auf 1,52 anstieg. Im Jahr 2009 lag die Durchschnittliche Perioden-Parität in Wien bei 1,49 verglichen mit dem Niveau von 1,57 für die anderen Bundesländer zusammen und von 1,54 für ganz Österreich. Im Vergleich dazu betrug die Perioden-TFR für Wien 1,36 und für Österreich als Ganzes 1,39. 


\section{Graphik 6}

Durchschnittliche Perioden-Parität (PAP) in Wien, im übrigen Bundesgebiet und in Österreich als Ganzes (1999-2009)

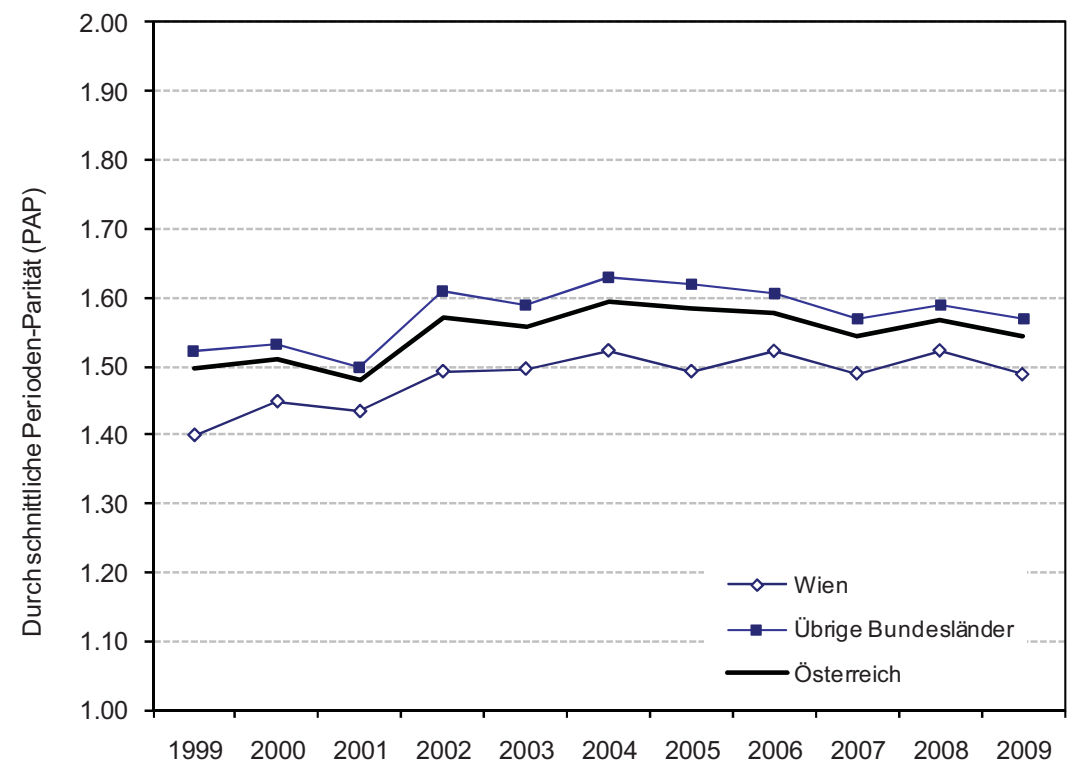

Quelle: Geburtenbarometer Wien, Berechnungen der Autoren

N.B.: Bruch in der Zeitreihe 2002 wegen der geänderten Verteilung von Frauen nach Alter und Parität auf Grund der Volkszählung 2001.

Die paritätsspezifischen Fertilitätsindikatoren zeigen, dass die Frauen in Wien etwas niedrigere Erstgeburtenziffern als jene in den anderen österreichischen Regionen aufweisen.

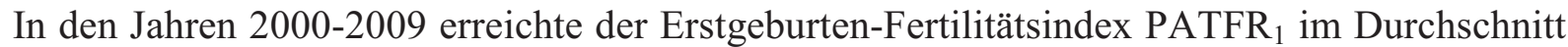
0,72 in Wien und 0,75 in Österreich, was eine Perioden-Kinderlosigkeitsrate von $28 \%$ in Wien und 25\% in Österreich andeutet (Graphik 7). ${ }^{11}$ Dies ist weitgehend in Übereinstimmung mit den beobachteten Tendenzen in der Kohorten-Paritätsverteilung, steht aber im Gegensatz zur Perioden-TFR für Erstgeburten, bei welcher der Wert für Wien durchwegs den für die anderen Bundesländer zusammen übertrifft (siehe oben). Da die Perioden-TFR stark durch Änderungen im Geburtentiming beeinflusst wird, ist die paritätsspezifische PATFR für Erstgeburten vertrauenswürdiger. Die Familienzuwachswahrscheinlichkeiten zeigen in Übereinstimmung mit den TFR-Indikatoren, dass die Frauen in Wien eine beträchtlich niedrigere Übergangsrate zur zweiten Geburt haben als die Frauen in den anderen Regionen Österreichs. Für 2000-2009 deuten die Perioden-PPRs an, dass drei Viertel der österreichischen Frauen mit einem Kind, die außerhalb Wiens lebten, eine zweite Entbindung haben würden, während nur zwei Drittel der Wienerinnen mit einem Kind letztendlich ein zweites bekommen würden. Im Gegensatz dazu übertreffen die Familienzuwachswahrscheinlichkeiten für dritte und vierte Kinder in Wien seit den frühen 2000er Jahren klar diejenigen in den anderen Bundesländern Österreichs: In den Jahren 2003-2009 betrug die PPR für dritte Geburten in Wien 0,40 und in Österreich ohne Wien 0,35; bei der PPR für die vierten Geburten war der Unterschied noch stärker ausgeprägt. Dieses Resultat bekräftigt die Feststellung bezüglich der steilen Zunahme

\footnotetext{
${ }^{11}$ Weil die Perioden-PATFR für Erstgeburten ebenfalls durch Tempo-Effekte auf Grund des Aufschiebens der Fertilität negativ beeinflusst ist - obschon viel weniger als die Perioden-TFR - ist es wahrscheinlich, dass die Kohorten-Kinderlosigkeit geringer sein wird. Für 1968 geborene Frauen wird die endgültige Kinderlosigkeit in der vorliegenden Studie für Wien auf 28\% und für Österreich ohne Wien auf $19 \%$ geschätzt.
} 
der TFR für Geburten dritter und höherer Ordnung in Wien am Ende der 1990er Jahre, wie oben berichtet (Graphik 5).

\section{Graphik 7}

Paritätsspezifischer Index der Erstgeburten 1991-2009 und Familienzuwachswahrscheinlichkeiten 1999-2009 in Wien und im übrigen Österreich
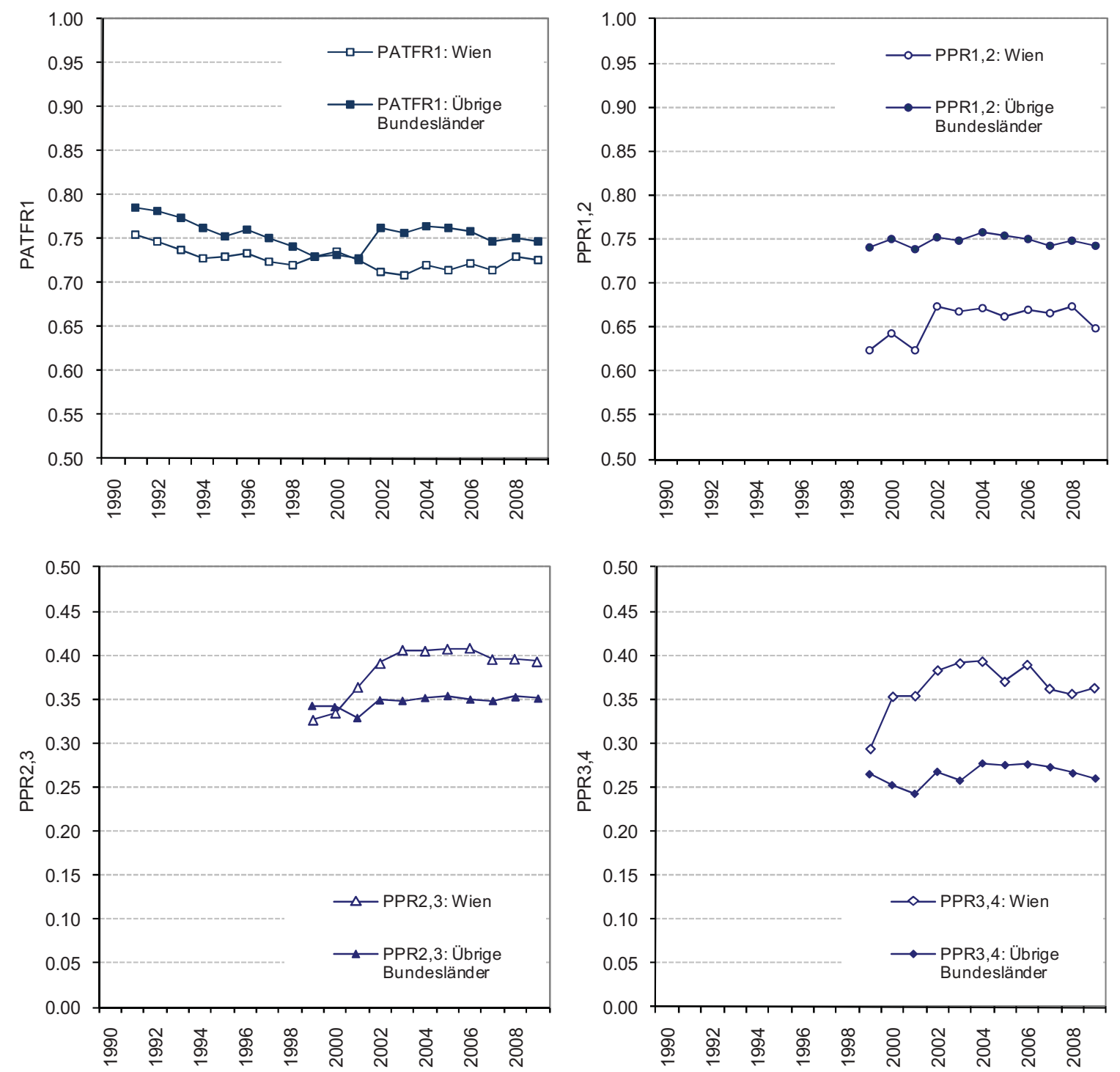

Quelle: Geburtenbarometer Wien, Berechnungen der Autoren

N.B.: Bruch in der Zeitreihe 2002 wegen der geänderten Verteilung von Frauen nach Alter und Parität auf Grund der Volkszählung 2001.

In Wien lebende Frauen sollten aufgrund ihrer höheren Ausbildung und umfangreicheren Karrieremöglichkeiten erwartungsgemäß Kinder später im Leben bekommen als Frauen in anderen Teilen Österreichs. Diese Erwartung wird für die Geburten insgesamt nicht bestätigt, bei denen die Tendenz im durchschnittlichen Fertilitätsalter in Wien und im übrigen Bundesgebiet fast identisch verlief, wobei sich das Durchschnittsalter von 1984 bis 2009 um drei Jahre erhöhte und 2009 29,6-29,7 Jahre erreichte. Mehr in Übereinstimmung mit dieser Hypothese ist die Beobachtung, dass die Wienerinnen ihr erstes Kind verhältnismäßig zu bekommen pflegten: 1984 hatten sie ihre erste Geburt im Durchschnitt mit 25,0 Jahren, ein Jahr später als die Frauen in den anderen Regionen $(23,9)$. Nach 1984 war die Verschiebung zu späte- 
rem Erstgeburten-Timing außerhalb Wiens jedoch ausgeprägter und regelmäßiger, und um 2000 gab es kaum noch einen Unterschied zwischen Wien und dem übrigen Bundesgebiet. 2009 bekamen die Wiener Frauen ihr erstes Kind durchschnittlich im Alter von 28,0 Jahren, nur 0,1 Jahre später als die Frauen in Österreich als Ganzes.

\section{Graphik 8}

Durchschnittliches Fertilitätsalter insgesamt und bei der Erstgeburt in Wien und im übrigen Österreich 1984-2009
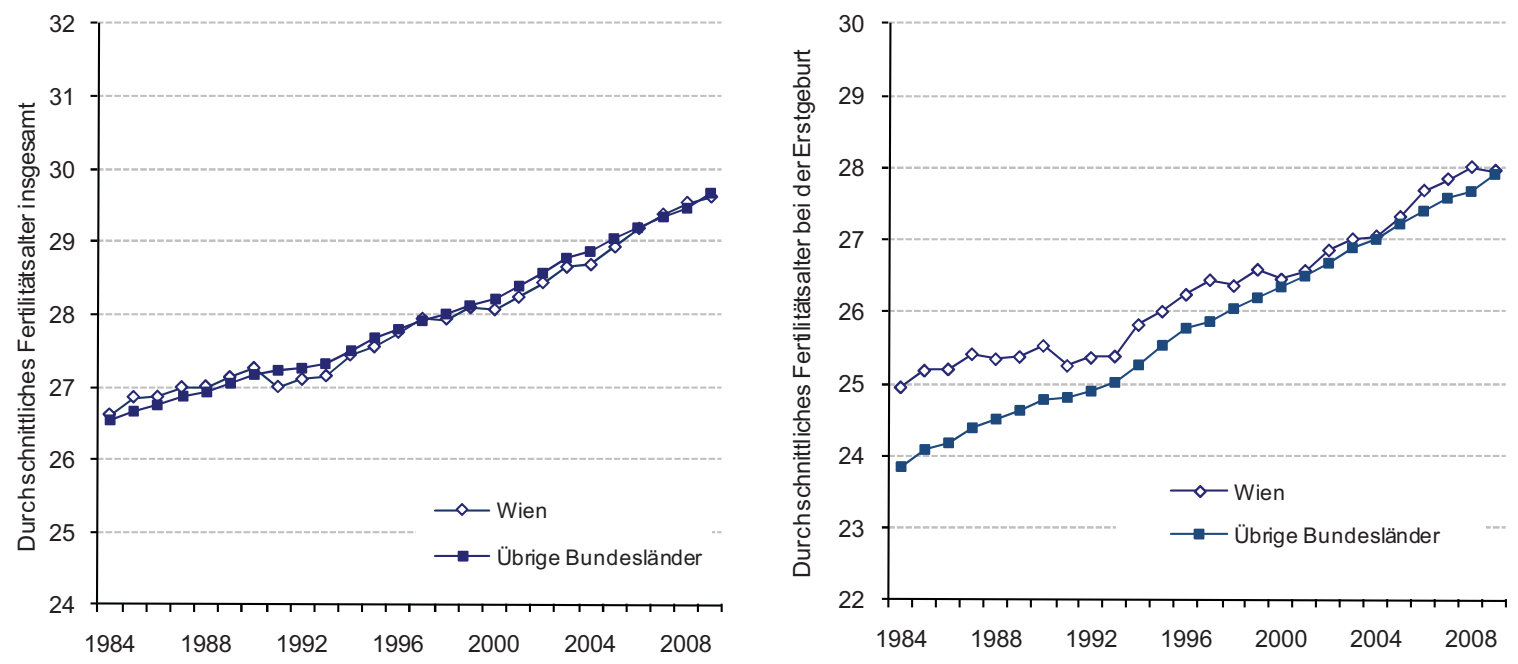

Quelle: Geburtenbarometer Wien, Berechnungen der Autoren

Die Trends im Durchschnittsalter verbergen jedoch eine beträchtliche Heterogenität in der Altersstruktur der Wiener Fertilität. Graphik 9 veranschaulicht diese Verschiedenartigkeit unter Verwendung der altersspezifischen Fruchtbarkeitsziffern und der Erstgeburtswahrscheinlichkeiten. 1984 stimmte das Muster der altersspezifischen Fertilitätsraten in Wien fast vollständig mit dem für das übrige Österreich überein. Im Jahr 2009 hatten die Wienerinnen erhöhte Fruchtbarkeitsziffern sowohl im niedrigeren (unter 23) als auch im höheren reproduktiven Alter (über 35) sowie einen viel weniger ausgeprägten Gipfel, der im Alter von 31 Jahren auftrat, ein Jahr später als in den anderen Regionen. Noch stärker ausgeprägt sind die Unterschiede im Altersmuster der Erstgeburtswahrscheinlichkeiten, die 2009 in Wien eine zweigipflige Kurve zeigen, mit einem starken Anstieg im sehr jungen Alter und einem lokalen Maximum bei 22 Jahren sowie einer deutlicheren Spitze im Alter von 32 Jahren. Demgegenüber ist die Altersverteilung der Erstgeburten in den übrigen Bundesländern zusammen mit nur einer Spitze bei 30 Jahre beträchtlich regelmäßiger. 


\section{Graphik 9a}

Altersspezifische Fruchtbarkeitsziffern in Wien und im übrigen Österreich 1984 und 2009
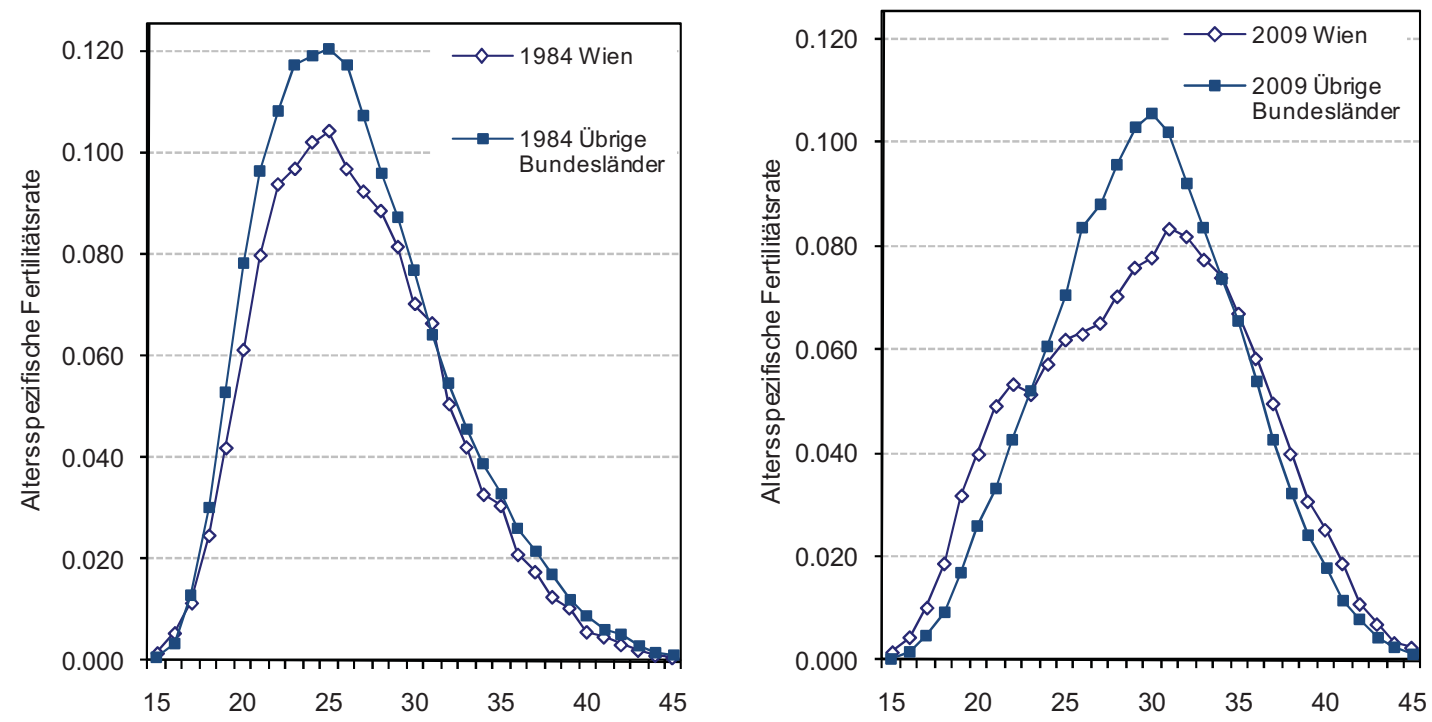

\section{Graphik 9b}

Altersspezifische Erstgeburtswahrscheinlichkeiten in Wien und im übrigen Österreich 1991 und 2009
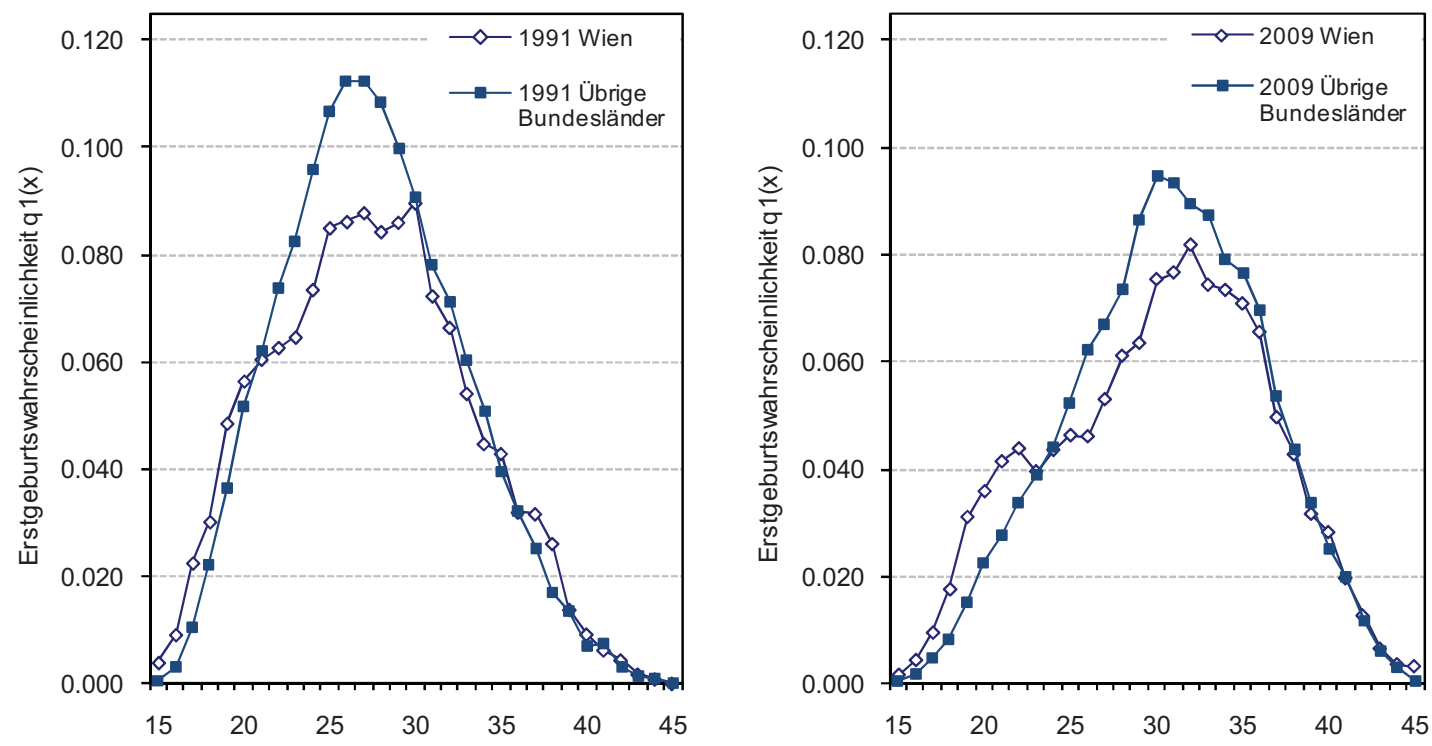

Quelle: Geburtenbarometer Wien, Berechnungen der Autoren

N.B.: Alter ist definiert als Differenz zwischen Jahreszahlen (im Kalenderjahr erreichtes Alter)

Um die steigende Altersdifferenzierung im Wiener Muster der Erstgeburten weiter zu veranschaulichen, liefert Tabelle 2 ausgewählte Indikatoren des Erstgeburten-Timings in Wien und im übrigen Österreich 1984 und 2009. In Wien stieg die Standardabweichung der Erstgeburtenziffern nach dem Alter zwischen 1984 und 2009 steil an und erreichte 6,2 Jahre, verglichen mit 5,3 Jahren für die anderen Bundesländer zusammen. 2009 erreichte der Anteil der Erstgeburtenziffern jenseits des Alters von 35 Jahren in Wien über 16\%, verglichen mit $11 \%$ im übrigen Österreich. Daten über die bedingten Erstgeburtswahrscheinlichkeiten zeigen, dass diese in Wien an den beiden Extremen der reproduktiven Lebensspanne viel höher 
sind als in den anderen Regionen, wobei Wienerinnen vor dem 20. und nach dem 40. Lebensjahr eine um 104\% beziehungsweise 13\% höhere Wahrscheinlichkeit einer ersten Geburt haben.

\section{Tabelle 2}

Ausgewählte Indikatoren des Erstgeburten-Timings in Wien und im übrigen Österreich 19842009

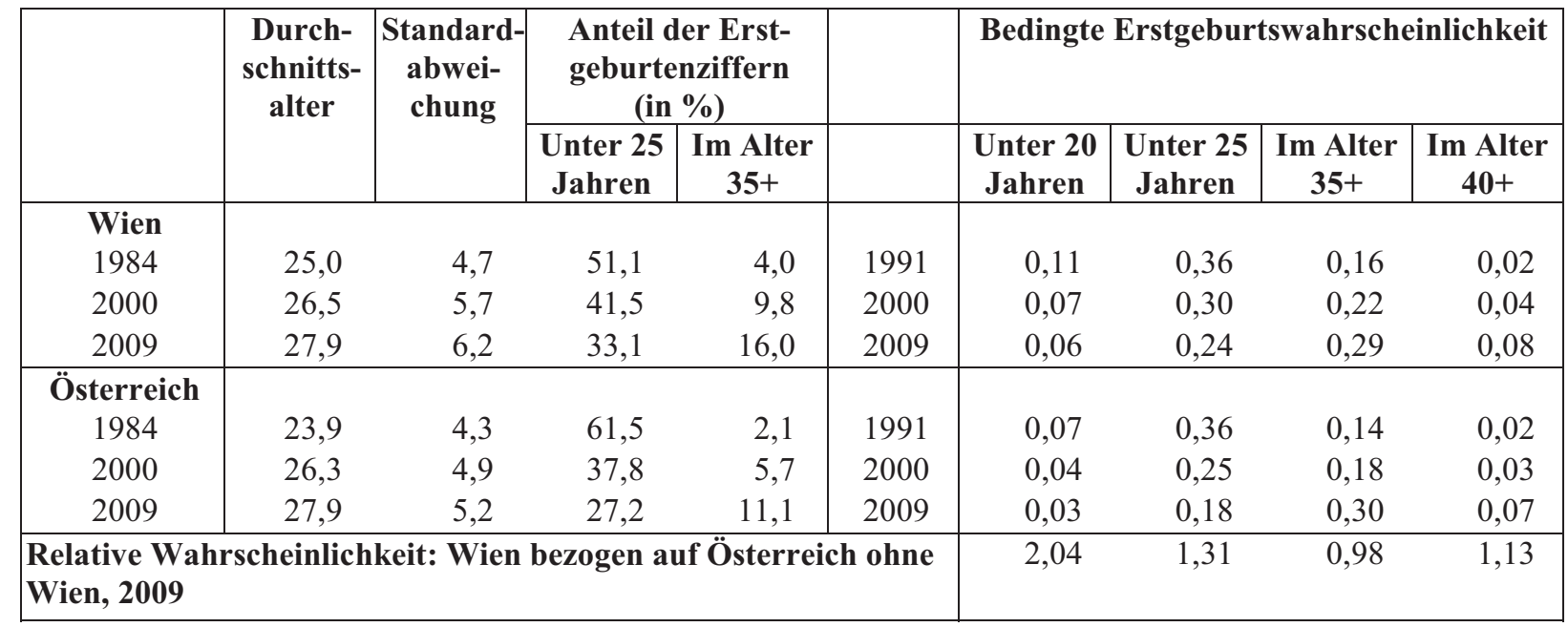

Quelle: Geburtenbarometer Wien, Berechnungen der Autoren

N.B.: Alle Indikatoren beziehen sich auf das im Kalenderjahr erreichte Alter (Differenz der Jahreszahlen); alle Frauen, die das Alter $\mathrm{x}$ während des Jahres $\mathrm{t}$ erreichen, werden daher das ganze Jahr hindurch als im Alter $\mathrm{x}$ stehend betrachtet, auch wenn ihr tatsächliches Alter (in vollendeten Jahren) im Zeitpunkt der Niederkunft x-1 war. Beispielsweise werden alle im Jahr 1980 geborenen Frauen während des ganzen Jahres 2008 als 28-jährig betrachtet.

\section{Tempo-Effekte in den Perioden-Fruchtbarkeitsziffern für Wien und Österreich}

Nach der Detailanalyse der jährlichen Fertilitätstrends in Wien wird nun die Aufmerksamkeit auf die Tempo-Effekte in der Periodenfertilität seit 1990 gelenkt; dabei wird die 1998 von Bongaarts und Feeney vorgeschlagene bereinigte (adjustierte) PeriodenGesamtfruchtbarkeitsrate (adjTFR) verwendet. Dieser Indikator kann eine grobe Schätzung der Größenordnung abgeben, um welche die TFR sowie die Durchschnitts-Periodenparität (PAP) durch den laufenden Aufschub der Geburt von Kindern auf spätere Lebensjahre niedergedrückt werden. Da die adjTFR für Wien durch sehr große Fluktuationen gekennzeichnet ist, fasst Tabelle 3 die Resultate für zwei ausgedehntere Zeiträume, 1990-99 und 2000-2006, zusammen, über welche diese jährlichen Fluktuationen ausgebügelt werden.

Die PAP kann als grober Näherungswert des Periodenfertilitätsniveaus benutzt werden, das nicht durch Tempo-Effekte verzerrt ist, die häufig die Perioden-TFR stark beeinflussen (Sobotka et al. 2005). Im Zeitraum 2000-2008 übertraf die PAP die Perioden-TFR in Wien um 0,11 und in Österreich um 0,17. Die tempoadjustierten TFRs nach Bongaarts und Feeney (1998) legen nahe, dass die negative Tempoverzerrung in der Perioden-TFR tatsächlich höher war, nämlich etwas über 0,25 für Wien und auch für Österreich. Tabelle 3 zeigt eine Konvergenz in den Fertilitätsniveaus zwischen Wien und Gesamtösterreich für 2000-2008 an; dabei erreichte die TFR durchschnittlich 1,38 und die adjTFR einen beträchtlich höheren Wert von 1,64. Ein geringer Unterschied blieb hinsichtlich der PAP, die in Wien um ungefähr 4\% niedriger lag als in Österreich insgesamt. 
Tabelle 3

Perioden-TFR, PAP und tempoadjustierte TFR in Wien und Österreich, 1990-99 und 20002008

\begin{tabular}{|l|rrr|c|}
\hline & TFR & PAP & adjTFR & $\begin{array}{c}\text { Geschätzter } \\
\text { Tempo-Effekt } \\
\text { (TFR-adjTFR) }\end{array}$ \\
\hline $\begin{array}{l}\text { Österreich } \\
1990-99\end{array}$ & 1,44 & & 1,64 & $-0,20$ \\
$2000-08$ & 1,39 & 1,55 & 1,64 & $-0,26$ \\
\hline Wien & 1,36 & & 1,51 & $-0,15$ \\
$1990-99$ & 1,38 & 1,49 & 1,64 & $-0,27$ \\
$2000-08$ & &
\end{tabular}

Quelle: Geburtenbarometer Wien, Berechnungen der Autoren

\section{Hauptergebnisse: Vierteljährliche Daten 2002-2010}

Im vorigen Abschnitt wurden Fruchtbarkeitsentwicklungen in Wien mit Hilfe der jährlichen Daten analysiert. In diesem Abschnitt wird ein kurzer Überblick über die vierteljährlichen Tendenzen in der Perioden-TFR, der PAP und den Familienzuwachswahrscheinlichkeiten 2002-2010 gegeben. Im Mittelpunkt steht Wien - diesmal ohne einen Vergleich mit anderen österreichischen Regionen, da die Resultate fast identisch mit den im vorhergehenden $\mathrm{Ab}$ schnitt besprochenen sind.

Nach Durchführung der Bereinigungen um jahreszeitliche und Kalenderfaktoren erwiesen sich die vierteljährlichen Fruchtbarkeitsindikatoren für Wien seit 2002 als ziemlich beständig. Während einige kurzfristige Veränderungen identifiziert werden können, besonders für die durchschnittliche Perioden-Parität, die zwischen 1,5 und 1,6 oszillierte, sind keine klaren längerfristigen Tendenzen oder Trendumschwünge zu bemerken, seit der jüngste Geburtenaufschwung um 2002 zu einem Ende kam. Eine ähnliche Stabilität in den Fertilitätstendenzen nach 2002 lässt sich auch in den Zeitreihen der österreichischen Daten beobachten (siehe z.B. Sobotka et al. 2005, Prskawetz et al. 2008). Die ökonomische Rezession von 2008-2009 hat in Wien keine sichtbare Markierung in den Zeittrends der Periodenfertilitätsindikatoren hinterlassen. Ein geringer Rückgang in den meisten Fertilitätsindikatoren im Jahr 2009 wurde anschließend durch einen Anstieg in den ersten drei Quartalen 2010 wettgemacht.

Die vierteljährlichen Zeitreihen der Familienzuwachswahrscheinlichkeiten zeigen kleinere Schwankungen, besonders für die dritten und vierten Geburten, die teils durch die verhältnismäßig geringen Zahlen bei solchen Geburten höherer Ordnung verursacht werden. Insgesamt sind die Zeitreihen jedoch bemerkenswert stabil, und besonders die vierteljährlichen Wahrscheinlichkeiten für die ersten und zweiten Geburten können leicht durch eine gerade Linie mit sehr wenig Veränderung über die Zeit approximiert werden. Die jüngste Rezession hatte keinen sichtbaren Effekt auf die Erstgeburtenziffern, die 2008 bis 2010 bei 0,72-0,74 lagen. Gleichzeitig zeigten die Familienzuwachswahrscheinlichkeiten für zweite Geburten zwischen dem ersten Quartal 2008 und dem zweiten Quartal 2009 einen leichten Rückgang von 0,69 auf 0,66, der in den ersten drei Vierteljahren 2010 von einem Anstieg auf das Niveau 0,71 abgelöst wurde (Graphik 10b). Die leichte Erhöhung der Periodenfertilität im Jahr 2010 
war demnach fast vollständig auf den Aufschwung in den Zweitgeburtenziffern zurückzuführen. $^{12}$

\section{Graphik 10a}

Vierteljährliche Reihe der Perioden-Gesamtfruchtbarkeitsziffer (TFR) und der durchschnittlichen Perioden-Parität (PAP) in Wien von 2002 bis zum dritten Quartal 2010

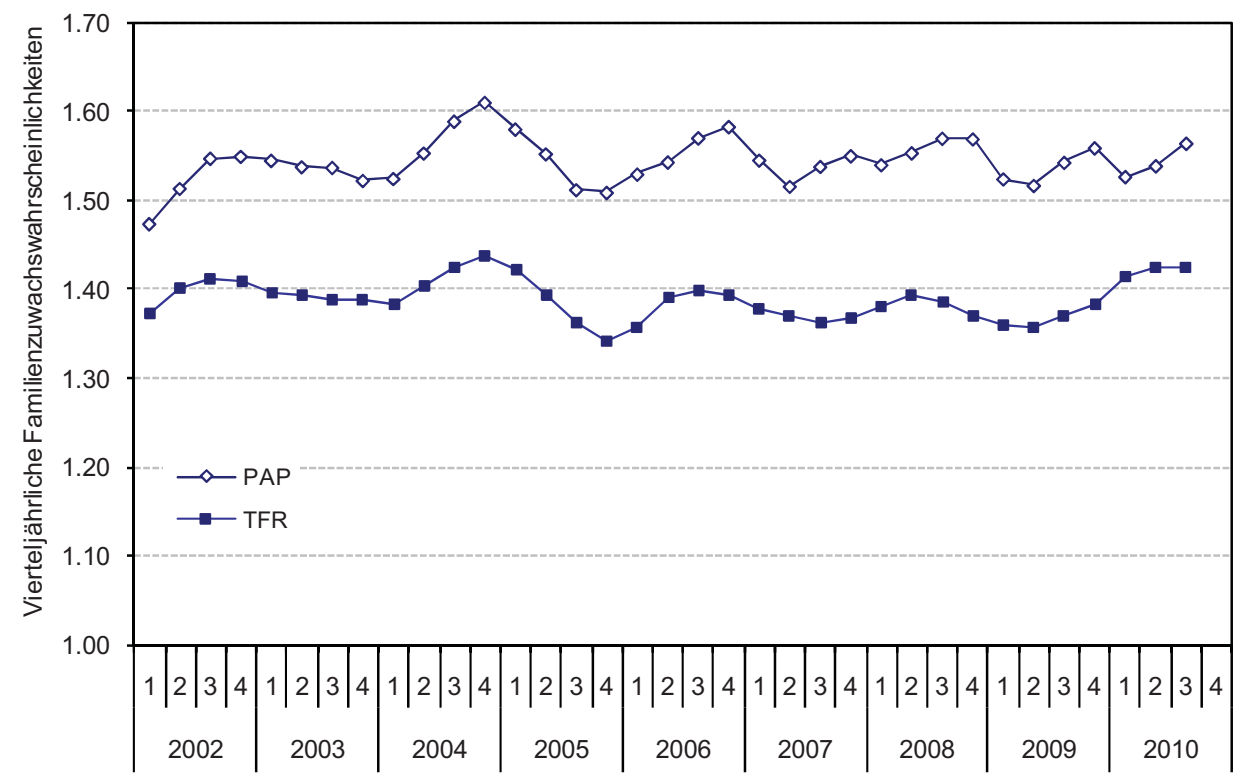

\section{Graphik 10b}

Vierteljährliche Reihe der Perioden-Familienzuwachswahrscheinlichkeiten $\left(\mathrm{PPR}_{\mathrm{i}, \mathrm{i}+1}\right)$ in Wien von 2002 bis zum dritten Quartal 2010

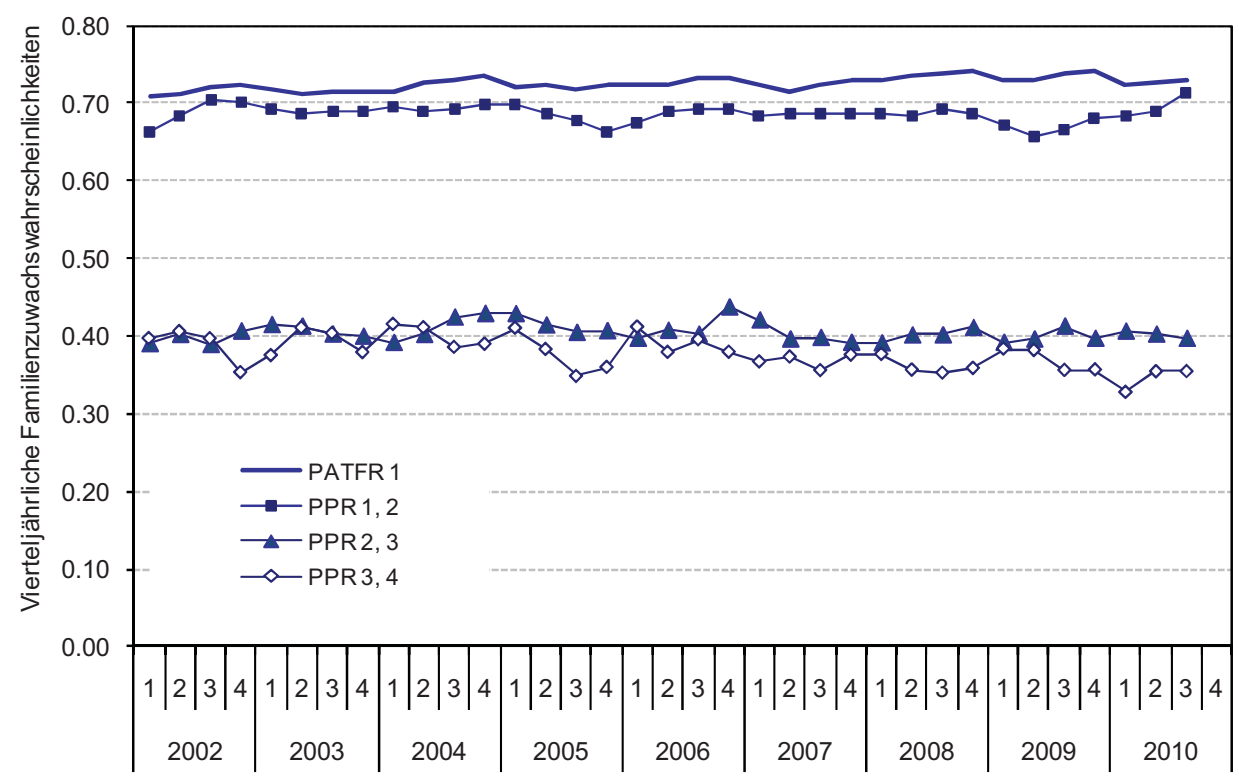

Quelle: Geburtenbarometer Wien, Berechnungen der Autoren

\footnotetext{
${ }^{12}$ Der seit September 2009 in Wien angebotene Gratisbesuch des Kindergartens für alle Altersstufen könnte einen Teil des daraufhin erfolgten Anstiegs der Rate zweiter Geburten erklären. Diese Möglichkeit muss jedoch sorgfältiger und unter Heranziehung einer längeren Zeitreihe untersucht werden.
} 


\section{Steigender Einfluss im Ausland geborener Frauen auf die Wiener Fertili- tätsmuster}

Die Erkenntnisse über den Anstieg der dritten und vierten Familienzuwachswahrscheinlichkeiten und die zunehmende Differenzierung in den Altersmustern der Geburtenraten in Wien sowie über die Konvergenz in der Fertilität zwischen Wien und dem übrigen Österreich weisen auf eine plausible Erklärung hin: Sie legen nahe, dass im Ausland geborene Frauen, gekennzeichnet durch ein unterschiedliches Fruchtbarkeitsverhalten, einen zunehmenden Einfluss auf die beobachteten Fertilitätstendenzen in Wien gehabt haben könnten. Dieser Abschnitt betrachtet den Anteil der Geburten von im Ausland geborenen Müttern, analysiert die unterschiedliche Fruchtbarkeit nach dem Herkunftsland und diskutiert die Auswirkung der im Ausland geborenen Frauen auf die Fertilitätsentwicklung in Wien.

\section{Graphik 11}

Prozentanteil der Lebendgeborenen von im Ausland geborenen Müttern in Wien, im übrigen Bundesgebiet und in Österreich insgesamt, 1984-2009

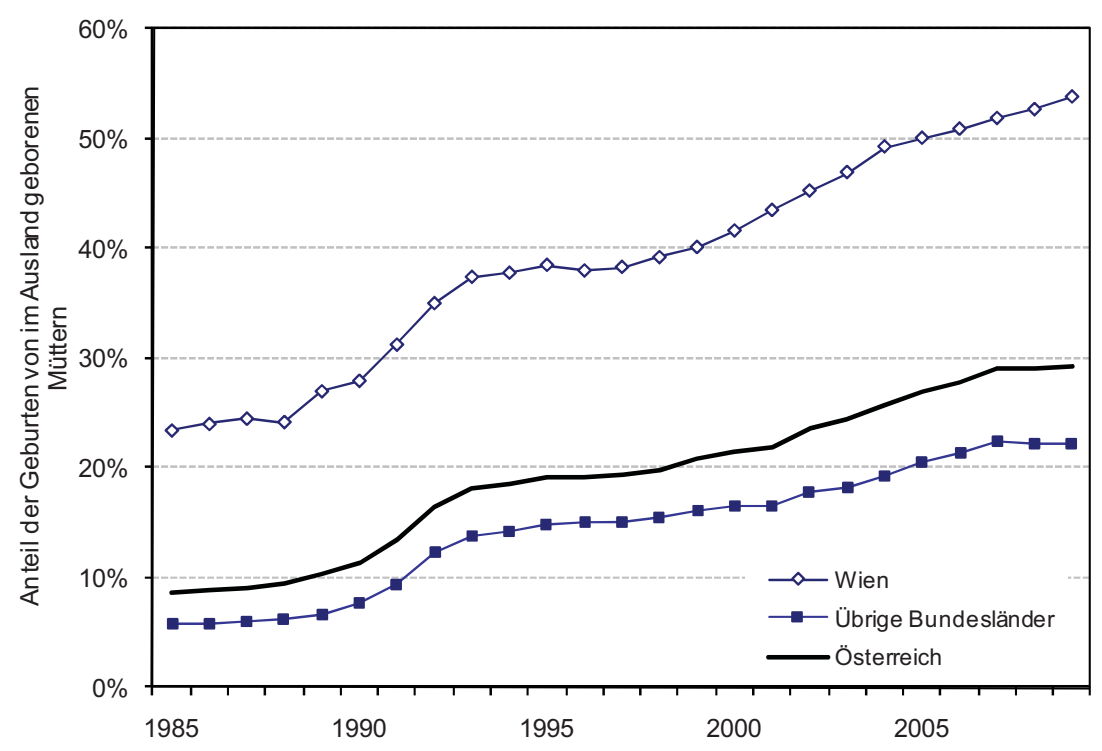

Quelle: Geburtenbarometer Wien, Berechnungen der Autoren

Graphik 11 zeigt eine beträchtliche Zunahme des Anteils der Geburten bei im Ausland geborenen Müttern in Wien seit der Mitte der 1980er Jahre. Bereits 1984 entfielen auf auslandsgebürtige Frauen 24\% aller Geburten Wiens, viermal so viele wie in den übrigen österreichischen Bundesländern. Ein kontinuierlicher, nur zur Mitte der 1990er kurz unterbrochener Anstieg im Prozentsatz der Neugeborenen von eingewanderten Frauen schob deren Anteil in Wien 2005 über die 50\%-Schwelle. 2009 stellten die im Ausland geborenen Frauen eine Majorität von 54\% der Geburten in Wien, verglichen mit 22\% in den übrigen Bundesländern und $29 \%$ in Österreich insgesamt. Dieser hohe Anteil ergibt sich durch eine Kombination der sehr niedrigen Geburtenziffern bei in Österreich geborenen Frauen und der hohen Zuwanderungsrate nach Wien aus dem Ausland, welche zur Zunahme der Anzahl im Ausland geborener Frauen im reproduktiven Alter führt. Die höhere Fruchtbarkeitsziffer der auslandsgebürtigen Frauen zeigt sich auch in ihrem hohen Anteil an den Neugeborenen dritter und höherer Ordnung in Wien: Während sie 2009 weniger als die Hälfte (49\%) der Erstgeburten stellten, erreichte ihr Beitrag 65\% der dritten Geburten und mehr als 70\% der Geburten vierter und höherer Ordnung (Anhang-Tabelle A7b). 
Seit dem Berichtsjahr 2008 ermöglichen die Daten eine Aufgliederung der Geburten nach dem Geburtsland der Mutter. Außer den aus Österreich stammenden Frauen, die 46\% aller Wiener Geburten zur Welt brachten, stellten 2009 die Frauen aus weiteren zwei Ländern, der Türkei und Serbien, ebenfalls beträchtliche Anteile von 10\% bzw. 6\% der Gesamtgeburten (wobei die Daten für Serbien auch die im Kosovo geborenen Frauen umfassten). Jeweils zwischen $2 \%$ und $4 \%$ trugen aus Bosnien-Herzegowina, Polen, Deutschland und Rumänien gebürtige Frauen bei, während die Neugeborenen von Frauen aus allen anderen Ländern in Wien 2009 ein Viertel ausmachten (Anhang-Tabelle A6b).

Die Analyse der Perioden-Gesamtfruchtbarkeitsziffern (TFR) 2002-2009 spezifiziert nach dem Geburtsland der Mutter ergibt einige wichtige Einblicke. ${ }^{13}$ Zunächst ist der Abstand zwischen der Fertilität der österreichgebürtigen und der auslandsgebürtigen Frauen sehr groß, besonders in Wien, wo die einheimischen Frauen 2009 die sehr niedrige TFR von 1,08 verzeichneten, während die TFR der im Ausland geborenen Frauen mit 1,82 um 68\% höher war (Graphik 12; siehe auch unten Tabelle 4). Dieser Kontrast ist nicht wegen der höheren Fertilität der im Ausland geborenen Frauen bemerkenswert, die verhältnismäßig niedrig ist, sondern wegen der in Wien beharrlich niedrigen Fertilität der inlandsgebürtigen Frauen, die um 0,2 niedriger ist als bei den in Österreich geborenen Frauen, die außerhalb Wiens leben. ${ }^{14}$ Demgegenüber ist der Abstand in der TFR zwischen den im Ausland geborenen Frauen, die in Wien und im übrigen Bundesgebiet leben, viel geringer.

Die in Österreich geborenen Frauen weisen ein stabiles Niveau der Periodenfertilität auf: Im Durchschnitt der Jahre 2002-2009 belief sich ihre Perioden-TFR auf 1,08 in Wien und 1,32 in den anderen Bundesländern zusammen. Dies kontrastiert mit einer rückläufigen Fruchtbarkeit bei den Immigrantinnen, insbesondere bei denen in Wien, deren TFR in dieser Zeit von 2,05 auf 1,82 fiel und so ihr Fertilitätsdifferenzial zu den einheimischen Frauen verringerte. Diese Zahlen verbergen jedoch eine beträchtliche Heterogenität in der Fruchtbarkeit verschiedener Gruppen von Eingewanderten, die für Österreich noch nicht analysiert wurde (für andere Länder siehe z.B. Coleman 1994, Haug et al. 2002, Sobotka 2008, Garssen und Nicolaas 2008). Im Vergleich zu den häufig publizierten Daten nach der Nationalität (Staatsangehörigkeit) der Mütter ist die TFR der auslandsgebürtigen Frauen in Österreich beträchtlich niedriger. Im Jahr 2008 hatten die Frauen mit ausländischer Staatsangehörigkeit eine TFR von 2.01 (Kytir und Wisbauer 2009: 876, Tabelle 8), wogegen die TFR von Frauen mit ausländischem Geburtsort (einschließlich jener mit österreichischer Staatsbürgerschaft) bei 1.90 notierte (siehe Tabelle A5).

\footnotetext{
${ }^{13}$ Fehlende Daten über die Paritätsverteilung der außerhalb Österreichs geborenen Frauen erlauben keine andere Berechnung von Fertilitätsindikatoren als die Perioden-TFR. Daher werden in diesem Abschnitt keine so ausgefeilten Fertilitätsindizes verwendet wie in anderen Teilen dieses Berichts.

${ }^{14}$ Ein derart niedriges Niveau der Periodenfertilitätsrate bei den in Österreich geborenen Wienerinnen könnte teilweise auch auf eine selektive Abwanderung aus Wien in umliegende suburbane Siedlungen unter jungen Österreicher/innen zurückzuführen sein, die eine größere Familie planen und ihr eigenes Haus oder Apartment in einer vermutlich familienfreundlicheren Umgebung außerhalb der Stadtgrenzen erwerben. Kulu (2006) zeigt, dass Binnen-Zugewanderte in kleineren Siedlungen Österreichs (Kleinstädte und ländliche Gebiete) höhere Fertilitätsraten aufweisen, während Zugezogene in Städte mit einer Bevölkerung über 100.000 nach der Wanderung eine sehr niedrige Fertilität haben.
} 


\section{Graphik 12}

Perioden-Gesamtfruchtbarkeitsziffer (TFR) von im Ausland und in Österreich geborenen Frauen in Wien und Österreich insgesamt, 2002-2009

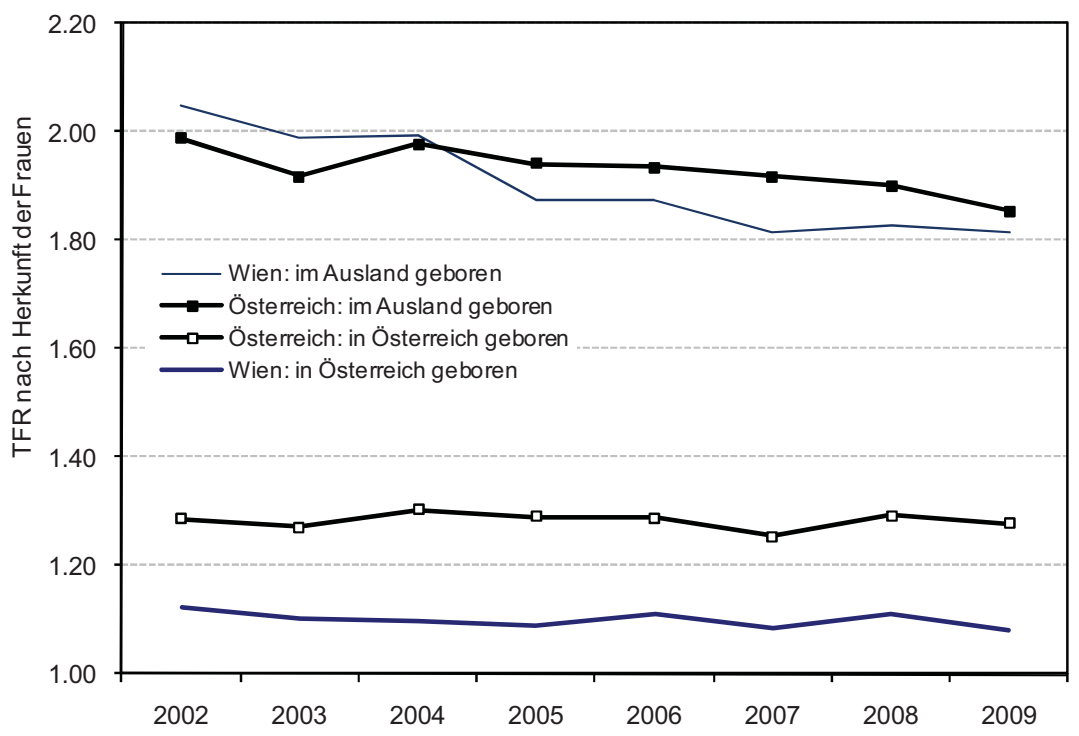

Quelle: Geburtenbarometer Wien, Berechnungen der Autoren

Die am stärksten ausgeprägten Fertilitätsunterschiede zwischen den im Inland und den im Ausland geborenen Frauen in Wien existieren in Bezug auf die Fruchtbarkeitsziffern dritter und höherer Ordnung. Die in Österreich geborenen Frauen wiesen 2002-2009 ein besonders niedriges Niveau der TFR dritter und höherer Ordnung auf, die bei 0,14 stabil war. Im Vergleich dazu hatten die im Ausland geborenen Frauen in Wien eine dreimal so hohe TFR3+, die dennoch in diesem Zeitraum sank, von 0,46 im Jahr 2002 auf 0,39 im Jahr 2009 (Graphik 13). Diese Daten legen nahe, dass der Aufwärtstrend bei den Fruchtbarkeitsziffern höherer Ordnung in Wien in den späten 1990er Jahren allein der höheren Fertilität der im Ausland geborenen Frauen zugeschrieben werden kann. Allerdings fehlt es für die Zeit vor 2002 an Daten für eine Rekonstruktion der Periodenfertilität nach dem Geburtsland der Mutter.

Der absolute Effekt der im Ausland geboren Frauen auf die Perioden-TFR kann einfach geschätzt werden, indem man eine Differenz zwischen der TFR für das ganze Land (Region) und der für die inlandsgebürtigen Frauen berechnet, die eine hypothetische TFR unter Ausschluss der Immigration repräsentiert (z.B. Sobotka 2008). Tabelle 4 zeigt, dass die eingewanderten Frauen in Wien einen erheblichen positiven Effekt auf die TFR haben, der sich 2002-2009 absolut auf rund 0,3 belief. Für das gesamte Bundesgebiet war der Nettobeitrag der Gewanderten viel niedriger und hob das österreichische TFR-Niveau 2009 um 0,11 an. 


\section{Graphik 13}

Perioden-TFR der Geburten dritter und höherer Ordnung von im Ausland bzw. in Österreich geborenen Frauen in Wien, 2002-2009

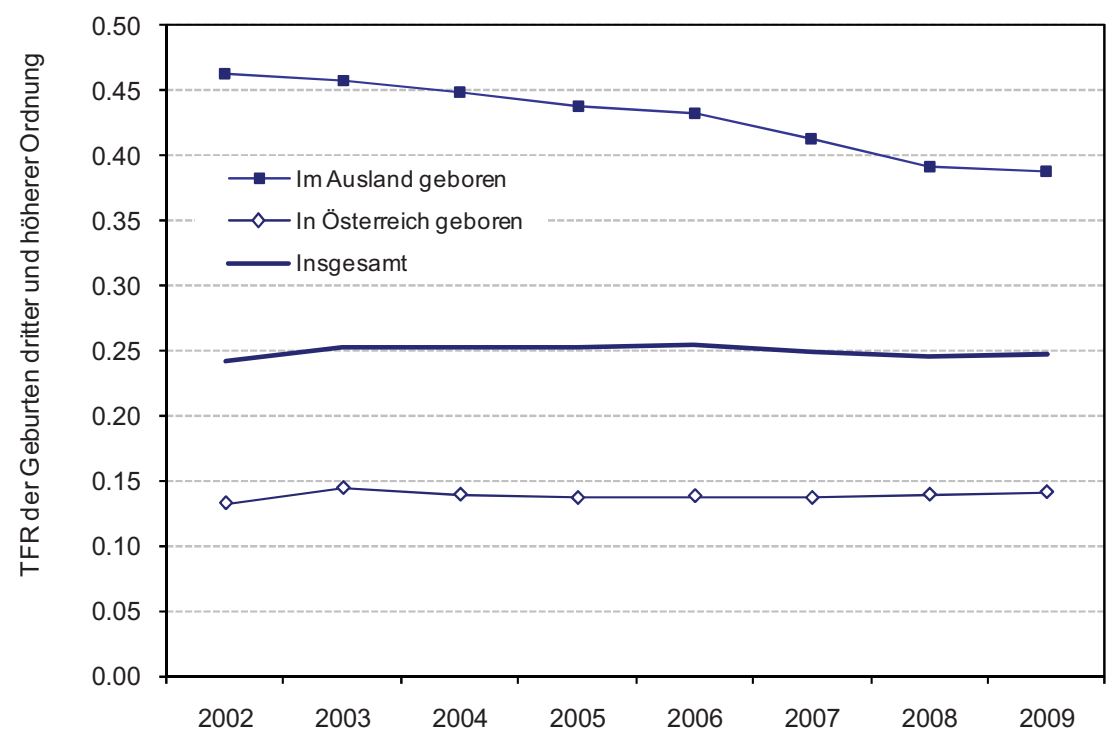

Quelle: Geburtenbarometer Wien, Berechnungen der Autoren

Tabelle 4

Perioden- Gesamtfruchtbarkeitsziffer (TFR) von im Inland bzw. im Ausland geborenen Frauen in Wien und Österreich sowie Netto-Effekt der Immigrantinnen auf die Perioden-TFR, 2002-2009

\begin{tabular}{|l|c|c|c|c|c|}
\hline & $\begin{array}{c}\text { TFR, in } \\
\text { Österreich } \\
\text { geborene } \\
\text { Frauen }\end{array}$ & $\begin{array}{c}\text { TFR, im } \\
\text { Ausland } \\
\text { geborene } \\
\text { Frauen }\end{array}$ & insgesamt & $\begin{array}{c}\text { TFR } \\
\text { Differenz in } \\
\text { Österreich vs. } \\
\text { im Ausland } \\
\text { Geborene }\end{array}$ & $\begin{array}{c}\text { Netto-Effekt der } \\
\text { Fertilität der } \\
\text { Immigrantinnen } \\
\text { auf die TFR } \\
\text { insgesamt }\end{array}$ \\
\hline Wien & 1,12 & 2,05 & 1,41 & 0,93 & 0,29 \\
2002 & 1,09 & 1,87 & 1,37 & 0,78 & 0,28 \\
2005 & 1,08 & 1,82 & 1,36 & 0,74 & 0,28 \\
2009 & 1,29 & 1,99 & 1,39 & & 0,70 \\
\hline Österreich & 1,29 & 1,94 & 1,41 & 0,65 & 0,11 \\
2002 & 1,28 & 1,85 & 1,39 & 0,58 & 0,12 \\
2005 & & & & & 0,11 \\
2009 &
\end{tabular}

Quelle: Geburtenbarometer Wien, Berechnungen der Autoren

Die Trends im Tempo der Fertilität (Geburtentiming) sind ebenfalls durch beträchtliche Unterschiede zwischen den in Österreich und den im Ausland geborenen Frauen gekennzeichnet. Obgleich beide Gruppen dazu neigen, ihre Erstgeburten in immer späterem Alter zu haben, schieben die eingewanderten Frauen das Kinderkriegen weniger stark hinaus und werden früher im Leben Mutter. In Wien macht der Unterschied im Durchschnittsalter beim ersten Kind zwischen inlands- und auslandsgebürtigen Frauen fast drei Jahre aus (Graphik 14). Zusätzlich bekommen die in Österreich geborenen Frauen ihr erstes Kind in Wien ungefähr ein Jahr später als in den anderen Bundesländern. Im Jahr 2009 belief sich das durchschnittliche Alter bei der Erstgeburt in Wien für alle Mütter auf 27,9 Jahre, wobei die Inland geborene Frauen mit 29,1 Jahren das Alter 29 überschritten, im Ausland geborene Mütter dagegen „nur“ 26,4 Jahre erreichten. 


\section{Graphik 14}

Durchschnittsalter bei der Erstgeburt für im Ausland bzw. in Österreich geborene Frauen in Wien und Österreich insgesamt, 2002-2009

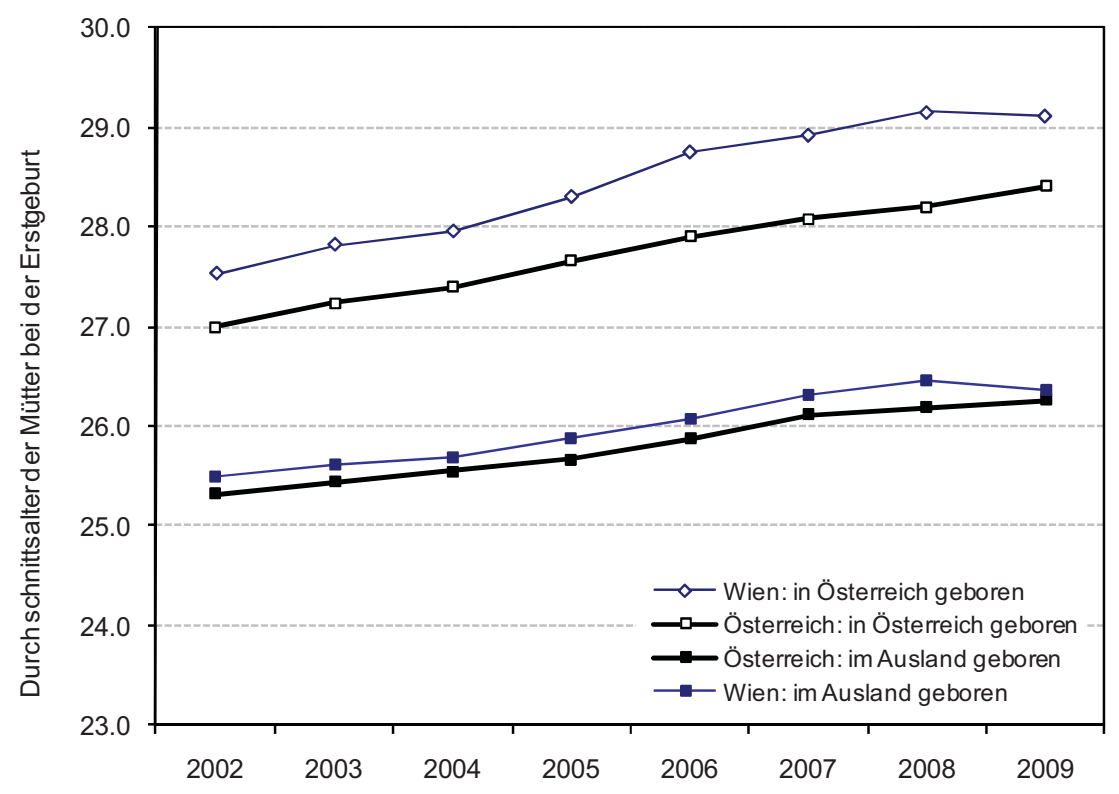

Quelle: Geburtenbarometer Wien, Berechnungen der Autoren

Die untererschiedlichen Altersmuster der Fruchtbarkeit in Wien für die in Österreich und die im Ausland geborenen Frauen werden weiters in Graphik 15 veranschaulicht. Ohne den Beitrag der Immigrantinnen wäre der „Fahrplan des Kinderkriegens“ in Wien beträchtlich „verspätet“ und würde sich deutlich von der Fertilitäts-Altersstruktur im übrigen Bundesgebiet unterscheiden (siehe Graphik 9a oben). Die Fertilitätsraten der im Ausland geborenen Frauen übertreffen die der „Einheimischen“ in Wien beträchtlich in jedem Alter bis 35 und sind im Alter von 19 bis 25 Jahren zweimal so hoch. Noch markantere Unterschiede gibt es im altersspezifischen Muster der Erstgeburtenziffern, die ihren jeweiligen Höhepunkt bei den auslandsgebürtigen Frauen im jungen Alter von 21 und bei den in Österreich geborenen Frauen mit späten 32 Jahren erreichen. Das Gesamtmuster der Wiener Erstgeburtenziffern, das keine ausgeprägte Spitze und zwei lokale Maxima in den Altern 21 und 30 hat, ist folglich ein Produkt der zwei ganz unterschiedlichen Familienbildungsmuster der in Österreich und der im Ausland geborenen Frauen. 


\section{Graphik 15}

Altersspezifische Fruchtbarkeitsziffern (Inzidenzraten) für alle Geburten (linkes Feld) und für Erstgeburten (rechtes Feld) der in Österreich und im Ausland geborenen Frauen in Wien 2009
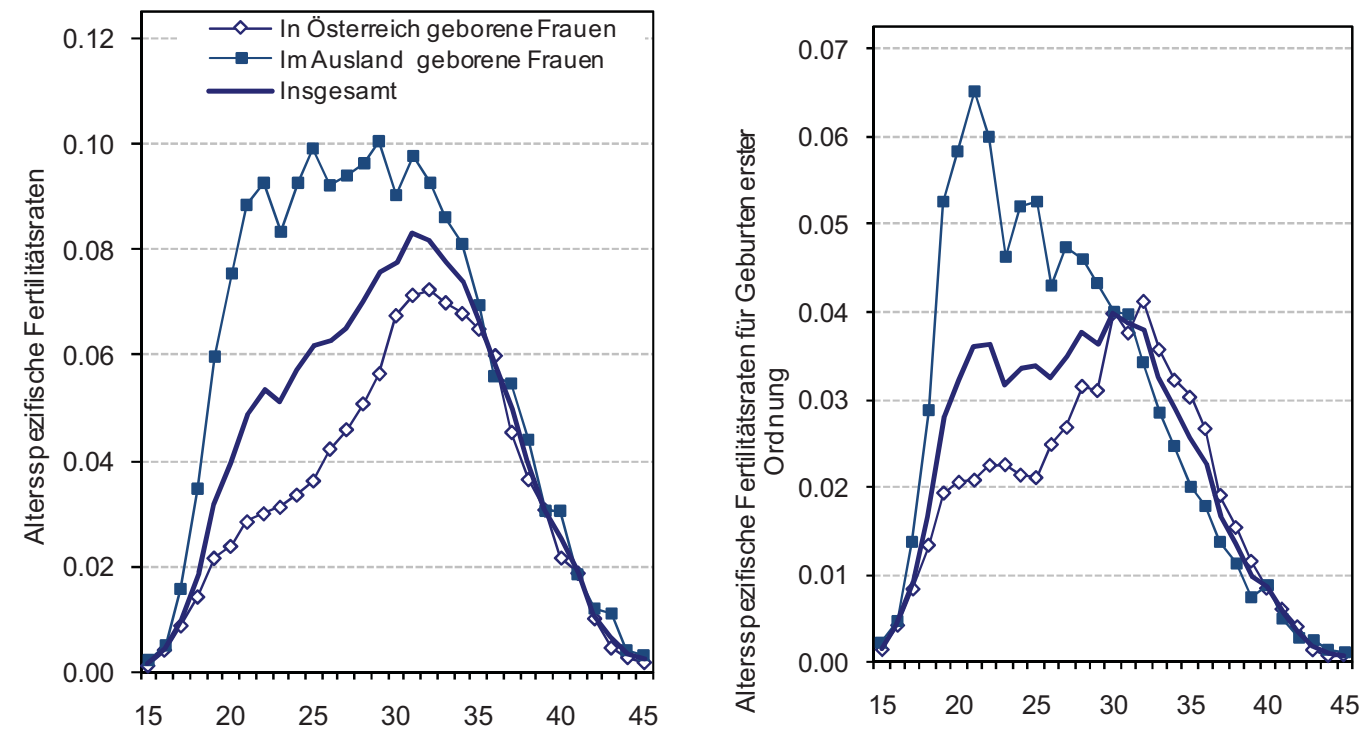

Quelle: Geburtenbarometer Wien, Berechnungen der Autoren

\section{Zusammenfassung und Schlussfolgerungen}

Diese Studie hat einen doppelten Zweck. Einerseits präsentiert er das neue Fertilitätsmonitoring für die Stadt (das Bundesland) Wien, welches dem früheren Beispiel des Geburtenbarometers für Österreich nachgebildet ist, und gibt einen Überblick über die verwendeten Daten, Methoden und Indikatoren sowie die Hauptergebnisse. Andererseits liefert er substanzielle Belege und eine Diskussion über Fertilitätstendenzen und -muster in Wien und kontrastiert sie mit den Daten für das übrige Bundesgebiet und Österreich als Ganzes. Weil die Fruchtbarkeit in Wien sich deutlich zwischen den „einheimischen“ (in Österreich geborenen) und den im Ausland geborenen (eingewanderten) Frauen unterscheidet, hat die vorliegende Analyse diesen Fertilitätsdifferenzialen und ihrem Einfluss auf die Muster der Gesamtfruchtbarkeit in Wien und Österreich Rechnung getragen. Unseres Wissens ist diese Studie die erste, in der Periodenfruchtbarkeitsziffern in Österreich nach dem Geburtsland der Mutter analysiert werden, obgleich nur die Möglichkeit bestand, sie für einen kurzen Zeitraum seit $2002 \mathrm{zu}$ rekonstruieren und nur zwischen den in Österreich und den im Ausland geborenen Frauen unterschieden werden konnte, ohne eine feinere Analyse nach Herkunftsländern zu geben. Bisher wurden in Untersuchungen über die Periodenfertilität in Österreich immer nur Daten analysiert, die sich auf die Nationalität (Staatsangehörigkeit) der Mutter beziehen. Diese geben ein verzerrtes Bild der Fertilitätsniveaus der Immigrantinnen und ihres Beitrags zur österreichischen Fertilität, weil viele Auslandsgebürtige während ihres langfristigen Aufenthalts hierzulande die österreichische Staatsbürgerschaft erhalten haben.

Während großer Teile des 20. Jahrhunderts lagen die Fruchtbarkeitsziffern in Wien auf Niveaus tief unterhalb der Fertilität in anderen Regionen Österreichs, und die PeriodenGesamtfertilitätsrate fiel in Wien mindestens viermal während des 20. Jahrhunderts auf „lowest low“ genannte Tiefststände unter 1,3: in der ersten Hälfte der 1930er Jahre, an der Wende der 40er und 50er Jahre, in der Mitte der 70er Jahre und noch einmal kurz 1997-99. Die extrem niedrige Fruchtbarkeit in der Vergangenheit führte zu einem frühen Einsetzen des 
schnellen Bevölkerungsalterns, zu hohen Raten der natürlichen Bevölkerungsabnahme und einer schrumpfenden Bevölkerung in den 1960er und 1970er Jahren (Lutz et al. 2003). Später jedoch kehrten sich die Wiener Bevölkerungstrends um, und 2005 hatte Wien eine wachsende Bevölkerung, steigende Geburtenzahlen, eine geringe Rate der natürlichen Bevölkerungszunahme, welche diejenige Österreichs übertraf, und ein Fertilitätsniveau, das zu dem des ganzen Landes konvergierte.

Die vorliegende Analyse der Wiener Fruchtbarkeit erbringt wichtige Einblicke, die wahrscheinlich auch für viele andere Großstädte Europas relevant sind. Es wird gezeigt, dass die Fertilitätsraten Wiens und Österreichs großteils wegen des massiven Fertilitätsrückgangs in Gesamtösterreich zwischen Mitte der 1960er und Mitte der 1980er Jahre konvergierten, kombiniert mit einer anfänglich langsameren Abnahme und später einer geringfügigen $\mathrm{Zu}-$ nahme der Fruchtbarkeitsziffern speziell in Wien. Diese Konvergenz manifestiert sich in den Zeitreihen der Perioden-Gesamtfertilitätsraten (TFR) und der durchschnittlichen PeriodenParität (PAP) - zwei regelmäßig im Geburtenbarometer berechneten Indikatoren - sowie in der tempobereinigten TFR, die darauf abzielt, die Effekte der Änderungen im Geburtentiming auf die Periodenfertilität herauszufiltern und die vor kurzem in Wien und Österreich gleichermaßen über 1,6 lag.

Die Fertilitätstendenzen in Wien werden in zunehmendem Maß von einer wachsenden Immigranten-Bevölkerung beeinflusst, die im Durchschnitt höhere Fruchtbarkeitsziffern aufweist und auf die derzeit mehr als Hälfte aller Geburten in Wien entfallen. Die vom Ausland zugewanderten Frauen in Wien hatten nicht nur seit Mitte der 1980er Jahre einen schnell wachsenden Anteil an den Gesamtgeburten und trugen so zu einer allmählichen Zunahme der absoluten Geburtenzahl in Wien bei, sondern ihre höheren Fruchtbarkeitsziffern halfen auch, die Periodenfertilität auf das Niveau der anderen Bundesländer (zusammen) anzuheben und, im Fall der Perioden-TFR, die Lücke zwischen Österreich und Wien zu schließen. Ihr NettoEffekt auf die Perioden-TFR in Wien belief sich 2002-2009 absolut auf 0,3 (Geburten pro Frau). Insbesondere haben die Eingewanderten beträchtlich höhere Raten bei dritten und weiteren Geburten und scheinen die wichtigste treibende Kraft beim Anstieg der Fruchtbarkeitsziffern höherer Ordnung in Wien in den späten 1990er Jahren und deren darauffolgenden Konvergenz zwischen Wien und Österreich insgesamt zu sein. Während Wien niedrigere Erst- und Zweitgeburtenziffern beibehält, übertreffen seine Familienzuwachswahrscheinlichkeiten für dritte und vierte Geburten jene für Österreich seit den frühen 2000er Jahren. Ohne Migration bliebe Wien eine Region mit weit niedrigeren Fruchtbarkeitsziffern als im übrigen Österreich sowie mit ununterbrochen fallenden Geburtenzahlen. Darüber hinaus bekommen die eingewanderten Frauen in Wien ihre Kinder viel früher als die in Österreich geborenen Frauen, was dementsprechend zur Streuung in den Altersmustern der Fertilität beiträgt.

In einer breiteren Perspektive kann Wien als Beispiel einer einzigartigen demographischen Entwicklung gesehen werden, eine Stadt mit hoher Kinderlosigkeit und niedriger Fruchtbarkeit, aber auch mit einer positiven Bilanz aus Geburten und Sterbefällen sowie anhaltendem Bevölkerungszuwachs, in der die Bevölkerungsentwicklung stark durch die direkten und (besonders durch Fertilität bedingten) indirekten Wirkungen der Immigration beeinflusst wird. Aus dem Fall Wien ergeben sich zwei wichtige Schlussfolgerungen für zeitgenössische entwickelte Länder. Erstens kann anhaltender Bevölkerungszuwachs durch intensive Zuwanderung sogar in Umgebungen mit einer langen Geschichte sehr niedriger Fertilität erzielt werden. Zweitens können Fruchtbarkeitsziffern, selbst wenn sie lange Zeit hindurch extrem niedrige Niveaus erreichen, zu höheren Niveaus zurückschwingen, entweder durch eine Zunahme der Fruchtbarkeit bei einheimischen Frauen oder durch einen allmählichen Ersatz 
von Bevölkerungen niedriger Fertilität durch fruchtbarere Gruppen, die aus anderen Regionen desselben Landes oder aus dem Ausland kommen können. Solche Szenarien können für viele wohlhabende Regionen zutreffen, die als Magneten der Immigration fungieren, einschließlich einiger in rascher Verstädterung befindlicher Entwicklungsländer, deren Städte ebenfalls sehr niedrige Fertilitätsniveaus erreicht haben. 


\section{Literatur}

Bongaarts, J. and G. Feeney. 1998. "On the quantum and tempo of fertility". Population and Development Review 24 (2): 271-291.

Coleman, D. 1994. "Trends in fertility and intermarriage among immigrant populations in Western Europe as measures of integration." Journal of Biosocial Science 26: 107-136.

Cottrel, A. 2004. Gnu regression, econometrics and time-series library-Gretl. [http://gretl.sourceforge.net].

Council of Europe. 1990. Cohort fertility in member states of the Council of Europe. Population Studies, No. 21, Strasbourg: Council of Europe.

Eurostat. 2009. Population and Social Conditions. Online database. [http://epp.eurostat.ec.europa.eu].

Garssen, J. and H. Nicolaas. 2008. "Fertility of Turkish and Moroccan women in the Netherlands: Adjustment to native level within one generation." Demographic Research 19(33): 1249-1280.

Gisser, R. et al. 1975. Die persönliche Situation der Frau. Volume 4 in: Bericht über die Situation der Frau in Österreich. Wien: Bundeskanzleramt.

Haug, W., P. Compton, and Y. Courbage (eds.). 2002. The Demographic Characteristics of Immigrant Populations. Population Studies, No. 38, Strasbourg: Council of Europe Publishing.

Jasilioniene, A., D.A. Jdanov, T. Sobotka, E.M. Andreev, K. Zeman, and V.M. Shkolnikov. 2010. Methods protocol for the Human Fertility Database (Version: 2 July 2010). [http://www.humanfertility.org/Docs/methods.pdf]

Kulu, H. 2006. "Fertility of internal migrants: comparison between Austria and Poland." Population, Space and Place 12(3): 147-170.

Kytir, J. and A. Wisbauer. 2009. "Demographische Strukturen und Trends 2008." Statistische Nachrichten 64 (10/2009): 869-884.

Lutz, W, S. Scherbov, and A. Hanika. 2003. “'Vienna: a city beyond aging' - revisited and revised" Vienna Yearbook of Population Research 2003: 181-195.

Prskawetz, A., T. Sobotka, I. Buber, R. Gisser, and H. Engelhardt. 2008. “Austria: persistent low fertility since the mid-1980s." Demographic Research 19(12): 293-360.

Rallu, L. and L. Toulemon. 1994. "Period fertility measures. The construction of different indices and their application to France, 1946-89". Population, An English Selection 6: 59-94.

Sobotka, T. 2008. "The rising importance of migrants for childbearing in Europe." Demographic Research 19 (Article 9): 225-248.

Sobotka, T., M. Winkler-Dworak, M. R. Testa, W. Lutz, D. Philipov, H. Engelhardt and R. Gisser. 2005. "Monthly Estimates of the Quantum of Fertility: Towards a Fertility Monitoring System in Austria". Vienna Yearbook of Population Research 2005: 109141.

Sobotka, T. and W. Lutz. 2009. "Misleading policy messages from the period TFR: Should we stop using it?" European Demographic Research Papers 4. Vienna: Vienna Institute of Demography. 
Spielauer, M. 2005. "Concentration of reproduction in Austria: General trends and differentials by educational attainment and urban-rural setting." Vienna Yearbook of Population Research 2005: 171-195.

Statistik Austria. 2005. Volkszählung 2001. Haushalte und Familien. Wien: Statistik Austria.

Statistik Austria. 2009a. Demographisches Jahrbuch Österreichs 2008. Wien: Statistik Austria.

Statistik Austria. 2009b. Demographische Indikatoren für Wien 1961-2008. Wien: Statistik Austria.

Statistik Austria. 2010. Demographische Jahrbuch Österreichs 2009. Wien: Statistik Austria.

VID. 2010. Geburtenbarometer : Monthly Monitoring of Fertility in Austria.

[http://www.oeaw.ac.at/vid/barometer/index.html]. 


\section{Appendix}

Tabelle A1: Zusammenfassende Indikatoren der Periodenfertilität in Österreich, Wien und Österreich ohne Wien, 1984-2009

\begin{tabular}{|c|c|c|c|c|c|c|c|c|c|c|c|c|}
\hline & \multicolumn{3}{|c|}{ Perioden- } & \multicolumn{3}{|c|}{ Durchschnittsalter der Mütter } & \multicolumn{3}{|c|}{ PATFR1 } & \multicolumn{3}{|c|}{ PAP } \\
\hline & Wien & $\begin{array}{c}\text { Öster- } \\
\text { reich }\end{array}$ & $\begin{array}{l}\text { Österreich } \\
\text { ohne Wien }\end{array}$ & Wien & $\begin{array}{c}\text { Öster- } \\
\text { reich }\end{array}$ & $\begin{array}{l}\text { Österreich } \\
\text { ohne Wien }\end{array}$ & Wien & $\begin{array}{c}\text { Öster- } \\
\text { reich }\end{array}$ & $\begin{array}{l}\text { Österreich } \\
\text { ohne Wien }\end{array}$ & Wien & $\begin{array}{c}\text { Öster- } \\
\text { reich }\end{array}$ & $\begin{array}{l}\text { Österreich } \\
\text { ohne Wien }\end{array}$ \\
\hline 1984 & 1,35 & 1,52 & 1,56 & 25,0 & 24,1 & 23,9 & & & & & & \\
\hline 1985 & 1,33 & 1,47 & 1,51 & 25,2 & 24,3 & 24,1 & & & & & & \\
\hline 1986 & 1,32 & 1,45 & 1,48 & 25,2 & 24,4 & 24,2 & & & & & & \\
\hline 1987 & 1,33 & 1,43 & 1,45 & 25,4 & 24,6 & 24,4 & & & & & & \\
\hline 1988 & 1,38 & 1,45 & 1,46 & 25,4 & 24,7 & 24,5 & & & & & & \\
\hline 1989 & 1,38 & 1,45 & 1,46 & 25,4 & 24,8 & 24,6 & & & & & & \\
\hline 1990 & 1,39 & 1,46 & 1,48 & 25,5 & 24,9 & 24,8 & & & & & & \\
\hline 1991 & 1,43 & 1,51 & 1,53 & 25,3 & 24,9 & 24,8 & 0,75 & 0,78 & 0,79 & & & \\
\hline 1992 & 1,42 & 1,50 & 1,53 & 25,4 & 25,0 & 24,9 & 0,75 & 0,77 & 0,78 & & & \\
\hline 1993 & 1,40 & 1,50 & 1,53 & 25,4 & 25,1 & 25,0 & 0,74 & 0,76 & 0,77 & & & \\
\hline 1994 & 1,38 & 1,47 & 1,49 & 25,8 & 25,4 & 25,3 & 0,73 & 0,75 & 0,76 & & & \\
\hline 1995 & 1,33 & 1,42 & 1,45 & 26,0 & 25,6 & 25,5 & 0,73 & 0,75 & 0,75 & & & \\
\hline 1996 & 1,34 & 1,45 & 1,47 & 26,2 & 25,9 & 25,8 & 0,73 & 0,75 & 0,76 & & & \\
\hline 1997 & 1,29 & 1,39 & 1,42 & 26,4 & 26,0 & 25,9 & 0,72 & 0,74 & 0,75 & & & \\
\hline 1998 & 1,29 & 1,37 & 1,39 & 26,4 & 26,1 & 26,1 & 0,72 & 0,74 & 0,74 & & & \\
\hline 1999 & 1,29 & 1,34 & 1,36 & 26,6 & 26,3 & 26,2 & 0,73 & 0,73 & 0,73 & 1,40 & 1,50 & 1,52 \\
\hline 2000 & 1,34 & 1,36 & 1,37 & 26,5 & 26,4 & 26,3 & 0,74 & 0,73 & 0,73 & 1,45 & 1,51 & 1,53 \\
\hline 2001 & 1,31 & 1,33 & 1,34 & 26,6 & 26,5 & 26,5 & 0,73 & 0,73 & 0,73 & 1,43 & 1,48 & 1,50 \\
\hline 2002 & 1,41 & 1,39 & 1,39 & 26,9 & 26,7 & 26,7 & 0,71 & 0,75 & 0,76 & 1,49 & 1,57 & 1,61 \\
\hline 2003 & 1,39 & 1,38 & 1,38 & 27,0 & 26,9 & 26,9 & 0,71 & 0,74 & 0,76 & 1,50 & 1,56 & 1,59 \\
\hline 2004 & 1,41 & 1,42 & 1,43 & 27,0 & 27,0 & 27,0 & 0,72 & 0,75 & 0,76 & 1,52 & 1,59 & 1,63 \\
\hline 2005 & 1,37 & 1,41 & 1,42 & 27,3 & 27,2 & 27,2 & 0,71 & 0,75 & 0,76 & 1,49 & 1,58 & 1,62 \\
\hline 2006 & 1,39 & 1,40 & 1,41 & 27,7 & 27,5 & 27,4 & 0,72 & 0,75 & 0,76 & 1,52 & 1,58 & 1,60 \\
\hline 2007 & 1,36 & 1,38 & 1,39 & 27,8 & 27,6 & 27,6 & 0,71 & 0,74 & 0,75 & 1,49 & 1,54 & 1,57 \\
\hline 2008 & 1,39 & 1,41 & 1,42 & 28,0 & 27,7 & 27,7 & 0,73 & 0,75 & 0,75 & 1,52 & 1,57 & 1,59 \\
\hline 2009 & 1,36 & 1,39 & 1,41 & 28,0 & 27,9 & 27,9 & 0,73 & 0,74 & 0,75 & 1,49 & 1,54 & 1,57 \\
\hline
\end{tabular}

Tabelle A2: Perioden-Gesamtfruchtbarkeitsziffer (Totale Fertilitätsrate, TFR) und Durchschnittsalter der Mütter bei der Geburt nach der Geburtenfolge in Wien, 1984-2009

\begin{tabular}{|c|c|c|c|c|c|c|c|c|c|c|c|c|}
\hline & \multicolumn{6}{|c|}{ TFR nach der Geburtenfolge } & \multicolumn{6}{|c|}{ Durchschnittsalter der Mütter bei der Geburt nach der Geburtenfolge } \\
\hline & 1 & 2 & 3 & 4 & $5+$ & Insgesamt & 1 & 2 & 3 & 4 & $5+$ & Insgesamt \\
\hline 1984 & 0,71 & 0,44 & 0,14 & 0,04 & 0,02 & 1,35 & 25,0 & 27,4 & 29,7 & 31,3 & 34,2 & 26,6 \\
\hline 1985 & 0,70 & 0,42 & 0,14 & 0,04 & 0,02 & 1,33 & 25,2 & 27,7 & 30,2 & 31,3 & 33,6 & 26,9 \\
\hline 1986 & 0,71 & 0,41 & 0,13 & 0,05 & 0,02 & 1,32 & 25,2 & 27,8 & 29,9 & 31,5 & 33,8 & 26,9 \\
\hline 1987 & 0,71 & 0,42 & 0,13 & 0,04 & 0,02 & 1,33 & 25,4 & 27,8 & 30,1 & 32,0 & 34,4 & 27,0 \\
\hline 1988 & 0,74 & 0,43 & 0,14 & 0,04 & 0,02 & 1,38 & 25,4 & 27,9 & 30,5 & 31,6 & 33,5 & 27,0 \\
\hline 1989 & 0,73 & 0,44 & 0,14 & 0,04 & 0,03 & 1,38 & 25,4 & 28,0 & 30,5 & 31,8 & 34,0 & 27,1 \\
\hline 1990 & 0,73 & 0,45 & 0,14 & 0,04 & 0,02 & 1,39 & 25,5 & 28,2 & 30,7 & 32,1 & 34,3 & 27,3 \\
\hline 1991 & 0,76 & 0,45 & 0,14 & 0,05 & 0,02 & 1,43 & 25,3 & 28,0 & 30,5 & 31,6 & 34,4 & 27,0 \\
\hline 1992 & 0,75 & 0,45 & 0,15 & 0,04 & 0,02 & 1,42 & 25,4 & 28,0 & 30,4 & 32,3 & 34,1 & 27,1 \\
\hline 1993 & 0,73 & 0,46 & 0,15 & 0,05 & 0,02 & 1,40 & 25,4 & 28,0 & 30,5 & 31,9 & 34,0 & 27,1 \\
\hline 1994 & 0,71 & 0,46 & 0,14 & 0,05 & 0,02 & 1,38 & 25,8 & 28,2 & 30,4 & 32,1 & 34,3 & 27,4 \\
\hline 1995 & 0,70 & 0,43 & 0,14 & 0,04 & 0,02 & 1,33 & 26,0 & 28,3 & 30,3 & 32,1 & 34,3 & 27,5 \\
\hline 1996 & 0,70 & 0,44 & 0,14 & 0,04 & 0,02 & 1,34 & 26,2 & 28,5 & 30,5 & 32,3 & 34,1 & 27,7 \\
\hline 1997 & 0,67 & 0,42 & 0,14 & 0,04 & 0,02 & 1,29 & 26,4 & 28,7 & 30,8 & 33,1 & 34,6 & 27,9 \\
\hline 1998 & 0,67 & 0,42 & 0,14 & 0,04 & 0,02 & 1,29 & 26,4 & 28,8 & 30,6 & 32,3 & 34,7 & 27,9 \\
\hline 1999 & 0,68 & 0,42 & 0,14 & 0,04 & 0,02 & 1,29 & 26,6 & 29,0 & 30,7 & 32,4 & 34,7 & 28,1 \\
\hline 2000 & 0,70 & 0,44 & 0,14 & 0,05 & 0,02 & 1,34 & 26,5 & 29,0 & 30,9 & 32,3 & 34,5 & 28,1 \\
\hline 2001 & 0,68 & 0,41 & 0,15 & 0,05 & 0,02 & 1,31 & 26,6 & 29,1 & 31,0 & 32,0 & 34,5 & 28,2 \\
\hline 2002 & 0,70 & 0,47 & 0,17 & 0,05 & 0,02 & 1,41 & 26,9 & 29,1 & 31,0 & 32,3 & 34,2 & 28,4 \\
\hline 2003 & 0,69 & 0,45 & 0,17 & 0,06 & 0,03 & 1,39 & 27,0 & 29,3 & 31,3 & 32,7 & 34,1 & 28,6 \\
\hline 2004 & 0,71 & 0,44 & 0,17 & 0,06 & 0,03 & 1,41 & 27,0 & 29,5 & 31,2 & 32,5 & 34,6 & 28,7 \\
\hline 2005 & 0,70 & 0,43 & 0,17 & 0,05 & 0,03 & 1,37 & 27,3 & 29,7 & 31,3 & 32,8 & 34,9 & 28,9 \\
\hline 2006 & 0,70 & 0,44 & 0,17 & 0,06 & 0,03 & 1,39 & 27,7 & 29,9 & 31,4 & 32,8 & 35,0 & 29,2 \\
\hline 2007 & 0,68 & 0,44 & 0,16 & 0,06 & 0,03 & 1,36 & 27,8 & 30,0 & 31,7 & 33,0 & 34,7 & 29,4 \\
\hline 2008 & 0,70 & 0,44 & 0,16 & 0,06 & 0,03 & 1,39 & 28,0 & 30,3 & 31,9 & 33,2 & 35,0 & 29,5 \\
\hline 2009 & 0,69 & 0,42 & 0,16 & 0,06 & 0,03 & 1,36 & 28,0 & 30,6 & 31,9 & 33,3 & 34,9 & 29,6 \\
\hline
\end{tabular}


Tabelle A3: Familienzuwachswahrscheinlichkeiten (Parity Progression Ratios, PPR) und Durchschnittliche Perioden-Parität (Period Average Parity, PAP) in Wien, 1999-2009

\begin{tabular}{|l|cccc|ccccc|c|}
\hline & \multicolumn{9}{|c|}{ Familienzuwachswahrscheinlichkeiten } & \multicolumn{3}{c|}{ Durchschnittliche Perioden-Parität (PAP) nach der Geburten- } \\
folge
\end{tabular}

Tabelle A4: Vierteljährliche Periodenfertilitätsindikatoren und Familienzuwachswahrscheinlichkeiten in Wien, 2002-2010

\begin{tabular}{|c|c|c|c|c|c|c|c|}
\hline Jahr & Quartal & TFR & PAP & PPR 0->1 & PPR 1->2 & PPR 2->3 & PPR 3->4 \\
\hline \multirow{4}{*}{2002} & I & 1,37 & 1,47 & 0,71 & 0,66 & 0,39 & 0,40 \\
\hline & II & 1,40 & 1,51 & 0,71 & 0,68 & 0,40 & 0,41 \\
\hline & III & 1,41 & 1,55 & 0,72 & 0,70 & 0,39 & 0,40 \\
\hline & IV & 1,41 & 1,55 & 0,72 & 0,70 & 0,41 & 0,35 \\
\hline \multirow[t]{4}{*}{2003} & I & 1,40 & 1,55 & 0,72 & 0,69 & 0,41 & 0,38 \\
\hline & II & 1,39 & 1,54 & 0,71 & 0,69 & 0,41 & 0,41 \\
\hline & III & 1,39 & 1,54 & 0,72 & 0,69 & 0,40 & 0,40 \\
\hline & IV & 1,39 & 1,52 & 0,72 & 0,69 & 0,40 & 0,38 \\
\hline \multirow[t]{4}{*}{2004} & I & 1,38 & 1,52 & 0,71 & 0,69 & 0,39 & 0,42 \\
\hline & II & 1,40 & 1,55 & 0,73 & 0,69 & 0,40 & 0,41 \\
\hline & III & 1,42 & 1,59 & 0,73 & 0,69 & 0,42 & 0,39 \\
\hline & IV & 1,44 & 1,61 & 0,73 & 0,70 & 0,43 & 0,39 \\
\hline \multirow[t]{4}{*}{2005} & I & 1,42 & 1,58 & 0,72 & 0,70 & 0,43 & 0,41 \\
\hline & II & 1,39 & 1,55 & 0,72 & 0,69 & 0,41 & 0,38 \\
\hline & III & 1,36 & 1,51 & 0,72 & 0,68 & 0,40 & 0,35 \\
\hline & IV & 1,34 & 1,51 & 0,72 & 0,66 & 0,41 & 0,36 \\
\hline \multirow[t]{4}{*}{2006} & I & 1,36 & 1,53 & 0,72 & 0,67 & 0,40 & 0,41 \\
\hline & II & 1,39 & 1,54 & 0,72 & 0,69 & 0,41 & 0,38 \\
\hline & III & 1,40 & 1,57 & 0,73 & 0,69 & 0,40 & 0,40 \\
\hline & IV & 1,39 & 1,58 & 0,73 & 0,69 & 0,44 & 0,38 \\
\hline \multirow[t]{4}{*}{2007} & I & 1,38 & 1,55 & 0,72 & 0,68 & 0,42 & 0,37 \\
\hline & II & 1,37 & 1,52 & 0,71 & 0,69 & 0,40 & 0,37 \\
\hline & III & 1,36 & 1,54 & 0,72 & 0,69 & 0,40 & 0,36 \\
\hline & IV & 1,37 & 1,55 & 0,73 & 0,69 & 0,39 & 0,38 \\
\hline \multirow[t]{4}{*}{2008} & $\mathbf{I}$ & 1,38 & 1,54 & 0,73 & 0,69 & 0,39 & 0,38 \\
\hline & II & 1,39 & 1,55 & 0,74 & 0,68 & 0,40 & 0,36 \\
\hline & III & 1,38 & 1,57 & 0,74 & 0,69 & 0,40 & 0,35 \\
\hline & IV & 1,37 & 1,57 & 0,74 & 0,69 & 0,41 & 0,36 \\
\hline \multirow[t]{4}{*}{2009} & I & 1,36 & 1,52 & 0,73 & 0,67 & 0,39 & 0,38 \\
\hline & II & 1,36 & 1,52 & 0,73 & 0,66 & 0,40 & 0,38 \\
\hline & III & 1,37 & 1,54 & 0,74 & 0,67 & 0,41 & 0,36 \\
\hline & IV & 1,38 & 1,56 & 0,74 & 0,68 & 0,40 & 0,36 \\
\hline \multirow[t]{3}{*}{2010} & I & 1,42 & 1,53 & 0,72 & 0,68 & 0,41 & 0,33 \\
\hline & II & 1,42 & 1,54 & 0,73 & 0,69 & 0,40 & 0,35 \\
\hline & III & 1,42 & 1,56 & 0,73 & 0,71 & 0,40 & 0,35 \\
\hline
\end{tabular}


Tabelle A5: Perioden-Gesamtfruchtbarkeitsziffer (TFR) sowie Durchschnittliches Fertilitätsalter insgesamt (DFA) und Durchschnittsalter der Mütter bei der bei der Erstgeburt (DAE) nach Geburtsland der Mutter, 2002-2009

\begin{tabular}{|c|c|c|c|c|c|c|c|c|c|}
\hline & \multicolumn{3}{|c|}{ Wien } & \multicolumn{3}{|c|}{ Österreich } & \multicolumn{3}{|c|}{ Österreich ohne Wien } \\
\hline & \multicolumn{3}{|c|}{ Geburtsland } & \multicolumn{3}{|c|}{ Geburtsland } & \multicolumn{3}{|c|}{ Geburtsland } \\
\hline & Österreich & Ausland & $\begin{array}{l}\text { Insge- } \\
\text { samt }\end{array}$ & reich & Ausland & Insgesamt & Österreich & Ausland & Insgesamt \\
\hline \multicolumn{10}{|l|}{ TFR } \\
\hline 2002 & 1,12 & 2,05 & 1,41 & 1,29 & 1,99 & 1,39 & 1,32 & 1,95 & 1,39 \\
\hline 2003 & 1,10 & 1,99 & 1,39 & 1,27 & 1,92 & 1,38 & 1,31 & 1,88 & 1,38 \\
\hline 2004 & 1,10 & 1,99 & 1,41 & 1,30 & 1,98 & 1,42 & 1,35 & 1,98 & 1,43 \\
\hline 2005 & 1,09 & 1,87 & 1,37 & 1,29 & 1,94 & 1,41 & 1,33 & 1,99 & 1,42 \\
\hline 2006 & 1,11 & 1,87 & 1,39 & 1,29 & 1,93 & 1,40 & 1,33 & 1,99 & 1,41 \\
\hline 2007 & 1,09 & 1,82 & 1,36 & 1,25 & 1,92 & 1,38 & 1,29 & 2,00 & 1,39 \\
\hline 2008 & 1,11 & 1,83 & 1,39 & 1,29 & 1,90 & 1,41 & 1,33 & 1,96 & 1,42 \\
\hline 2009 & 1,08 & 1,82 & 1,36 & 1,28 & 1,85 & 1,39 & 1,32 & 1,89 & 1,41 \\
\hline \multicolumn{10}{|l|}{ DFA } \\
\hline 2002 & 28,8 & 27,8 & 28,4 & 28,7 & 27,8 & 28,6 & 28,7 & 27,7 & 28,6 \\
\hline 2003 & 29,1 & 27,9 & 28,6 & 28,9 & 28,0 & 28,8 & 28,9 & 28,0 & 28,8 \\
\hline 2004 & 29,3 & 27,8 & 28,7 & 29,0 & 27,9 & 28,8 & 29,0 & 28,0 & 28,8 \\
\hline 2005 & 29,5 & 28,2 & 28,9 & 29,3 & 28,1 & 29,0 & 29,2 & 28,0 & 29,0 \\
\hline 2006 & 29,8 & 28,3 & 29,2 & 29,5 & 28,2 & 29,2 & 29,4 & 28,1 & 29,2 \\
\hline 2007 & 30,1 & 28,4 & 29,4 & 29,7 & 28,3 & 29,4 & 29,6 & 28,2 & 29,3 \\
\hline 2008 & 30,3 & 28,5 & 29,5 & 29,8 & 28,4 & 29,5 & 29,7 & 28,2 & 29,5 \\
\hline 2009 & 30,5 & 28,5 & 29,6 & 30,0 & 28,4 & 29,7 & 29,9 & 28,3 & 29,7 \\
\hline \multicolumn{10}{|l|}{ DAE } \\
\hline 2002 & 27,5 & 25,5 & 26,9 & 27,0 & 25,3 & 26,7 & 26,9 & 25,1 & 26,7 \\
\hline 2003 & 27,8 & 25,6 & 27,0 & 27,2 & 25,4 & 26,9 & 27,1 & 25,3 & 26,9 \\
\hline 2004 & 28,0 & 25,7 & 27,0 & 27,4 & 25,5 & 27,0 & 27,3 & 25,4 & 27,0 \\
\hline 2005 & 28,3 & 25,9 & 27,3 & 27,7 & 25,7 & 27,2 & 27,5 & 25,5 & 27,2 \\
\hline 2006 & 28,8 & 26,1 & 27,7 & 27,9 & 25,9 & 27,5 & 27,7 & 25,7 & 27,4 \\
\hline 2007 & 28,9 & 26,3 & 27,8 & 28,1 & 26,1 & 27,6 & 27,9 & 26,0 & 27,6 \\
\hline 2008 & 29,2 & 26,5 & 28,0 & 28,2 & 26,2 & 27,7 & 28,0 & 26,0 & 27,7 \\
\hline 2009 & 29,1 & 26,4 & 27,9 & 28,4 & 26,3 & 27,9 & 28,3 & 26,2 & 27,9 \\
\hline
\end{tabular}

Tabellen A6a und 6b: Anteil der Lebendgeborenen nach dem Geburtsland der Mutter, Österreich und Wien, 2009 (Top Ten)

\begin{tabular}{|l|r|}
\hline Geburtsland & Österreich \\
\hline Österreich & $70,7 \%$ \\
\hline Türkei & $5,6 \%$ \\
Bosnien und Herzegowina & $3,2 \%$ \\
Deutschland & $2,9 \%$ \\
Serbien & $2,1 \%$ \\
Rumänien & $1,6 \%$ \\
Russland & $1,3 \%$ \\
Kosovo & $1,3 \%$ \\
Polen & $1,1 \%$ \\
Slowakei & $0,7 \%$ \\
\hline Sonstige & $9,5 \%$ \\
\hline
\end{tabular}

\begin{tabular}{|l|r|}
\hline Geburtsland & Wien \\
\hline Österreich & $46,2 \%$ \\
\hline Türkei & $10,4 \%$ \\
Serbien & $6,1 \%$ \\
Bosnien und Herzegowina & $3,8 \%$ \\
Polen & $3,4 \%$ \\
Deutschland & $3,0 \%$ \\
Rumänien & $2,4 \%$ \\
Russland & $1,9 \%$ \\
Slowakei & $1,7 \%$ \\
Kosovo & $1,6 \%$ \\
\hline Sonstige & $19,7 \%$ \\
\hline
\end{tabular}


Tabelle A7a: Verteilung der Lebendgeborenen in Wien und Österreich von in Österreich und im Ausland geborenen Müttern nach der Geburtenfolge 2009

\begin{tabular}{|l|rrr|rrr|}
\hline & \multicolumn{3}{|c|}{ Wien } & \multicolumn{3}{c|}{ Österreich } \\
\hline \multirow{3}{*}{ Geburtenfolge } & \multicolumn{2}{|c|}{ Geburtsland der Mutter } & \multicolumn{2}{c|}{ Geburtsland der Mutter } \\
\cline { 2 - 7 } & Österreich & Ausland & Insgesamt & Österreich & Ausland & Insgesamt \\
\hline $\mathbf{1}$ & $55 \%$ & $45 \%$ & $50 \%$ & $49 \%$ & $43 \%$ & $47 \%$ \\
$\mathbf{2}$ & $31 \%$ & $32 \%$ & $31 \%$ & $36 \%$ & $33 \%$ & $35 \%$ \\
$\mathbf{3}$ & $9 \%$ & $15 \%$ & $12 \%$ & $12 \%$ & $15 \%$ & $13 \%$ \\
$\mathbf{4}$ & $3 \%$ & $6 \%$ & $4 \%$ & $3 \%$ & $6 \%$ & $4 \%$ \\
$\mathbf{5}+$ & $2 \%$ & $3 \%$ & $2 \%$ & $1 \%$ & $3 \%$ & $2 \%$ \\
\hline Insgesamt & $\mathbf{1 0 0} \%$ & $\mathbf{1 0 0} \%$ & $\mathbf{1 0 0} \%$ & $\mathbf{1 0 0} \%$ & $\mathbf{1 0 0 \%}$ & $\mathbf{1 0 0} \%$ \\
\hline
\end{tabular}

Tabelle A7b: Anteil der Lebendgeborenen in Wien und Österreich nach der Geburtenfolge und dem Geburtsland der Mutter 2009

\begin{tabular}{|l|ccc|crc|}
\hline \multirow{3}{*}{ Geburtenfolge } & \multicolumn{3}{|c|}{ Wien } & \multicolumn{3}{c|}{ Österreich } \\
\cline { 2 - 7 } & \multicolumn{2}{|c|}{ Geburtsland der Mutter } & \multicolumn{2}{c|}{ Geburtsland der Mutter } \\
\hline \multirow{2}{*}{$\mathbf{2}$} & Österreich & Ausland & Insgesamt & Österreich & Ausland & Insgesamt \\
$\mathbf{2}$ & $51 \%$ & $49 \%$ & $\mathbf{1 0 0} \%$ & $73 \%$ & $27 \%$ & $\mathbf{1 0 0} \%$ \\
$\mathbf{3}$ & $46 \%$ & $54 \%$ & $\mathbf{1 0 0} \%$ & $73 \%$ & $27 \%$ & $\mathbf{1 0 0} \%$ \\
$\mathbf{5}+$ & $35 \%$ & $65 \%$ & $\mathbf{1 0 0} \%$ & $65 \%$ & $35 \%$ & $\mathbf{1 0 0} \%$ \\
\hline Insgesamt & $30 \%$ & $70 \%$ & $\mathbf{1 0 0} \%$ & $56 \%$ & $44 \%$ & $\mathbf{1 0 0} \%$ \\
\hline
\end{tabular}




\section{VIENNA INSTITUTE OF DEMOGRAPHY}

\section{Working Papers}

Feichtinger Gustav, Michael Kuhn, Alexia Prskawetz, and Stefan Wrzaczek, The Reproductive Value as Part of the Shadow Price of Population, VID Working Paper 06/2011.

Barakat, Bilal, Time is Money: Could Deferred Graduate Retirement Finance Higher Education?, VID Working Paper 05/2011.

Riosmena, Fernando, Maria Winkler-Dworak, Alexia Prskawetz, and Gustav Feichtinger, The Impact of Policies Influencing the Demography of Age Structured

Populations: Lessons from Academies of Science, VID Working Paper 04/2011.

Buber, Isabella and Katrin Fliegenschnee, Are you Ready for a Child? A Methodological Triangulation on Fertility Intentions in Austria, VID Working Paper $03 / 2011$.

Fent, Thomas, Belinda Aparicio Diaz, and Alexia Prskawetz, Family Policies in the Context of Low Fertility and Social Structure, VID Working Paper 02/2011.

Buber, Isabella, Caroline Berghammer, and Alexia Prskawetz, Doing Science, Forgoing Childbearing? Evidence from a Sample of Female Scientists in Austria, VID Working Paper 01/2011.

Strulik, Holger, Klaus Prettner, and Alexia Prskawetz, R\&D-Based Growth in the Post-Modern Era, VID Working Paper 09/2010.

Prettner, Klaus and Alexia Prskawetz, Demographic Change in Models of Endogenous Economic Growth. A Survey, VID Working Paper 08/2010.

Flandorfer, Priska, Christian Wegner, and Isabella Buber, Gender Roles and Smoking Behaviour, VID Working Paper 07/2010.

Barakat, Bilal, Johannes Holler, Klaus Prettner, and Julia Schuster, The Impact of the Economic Crisis on Labour and Education in Europe, VID Working Paper 06/2010.

Grafeneder-Weissteiner, Theresa and Klaus Prettner, Agglomeration Processes in Ageing Societies, VID Working Paper 05/2010.

Buber, Isabella, Parity-specific weights for the Austrian Generations and Gender Survey, VID Working Paper 04/2010.

The Vienna Institute of Demography Working Paper Series receives only limited review. Views or opinions expressed herein are entirely those of the authors. 